Acta Botanica Brasilica - 35(3): 323-338. July-September 2021.

\title{
Elucidating plant-pollinator interactions in South Brazilian grasslands: What do we know and where are we going?
}

\section{Suiane Santos Oleques ${ }^{1 *}$ (D), Tatiana Teixeira de Souza-Chies, 2 (1) and Rubem Samuel de Avila Jr ${ }^{3}$ (i)}

Received: May 13, 2020

Accepted: March 22, 2021

\begin{abstract}
Grassland ecosystems present patterns of plant-pollinator interactions that may be linked to habitat heterogeneity, plant composition and disturbances. Most studies about plant-pollinator interactions in the Neotropics were conducted in forest, savanna-like, or Andean vegetation. However, the current increase in the number of studies about interactions in grassland vegetation promises a better understanding of the pollination ecology of these landscapes. In this systematic review, we summarised information from 24 articles about plant-pollinator interactions in South Brazilian grasslands. We highlighted patterns of plant-pollinator interactions, indicating their particularities compared to other grassland communities in South America. Bees are important pollinators of many plant species in these grasslands and most plants are visited by more than one group of pollinators. Among the plant species visited by a single pollinator group, most were visited by bees. However, many types of pollinators, plant species, habitats, and regions have, thus far, received little sampling effort. Pollination by groups other than bees, such as nocturnal pollinators, flies, beetles, and birds, is particularly understudied. The information provided in this review summarizes data that could be used to foster more detailed pollination studies to understand the diversification and maintenance of grassland floras of South Brazil.
\end{abstract}

Keywords: Atlantic Rain Forest, bee-pollination, grassland vegetation, mutualistic interaction, Pampa, pollination systems, subtropical grasslands

1 Programa de Pós-graduação em Botânica, Universidade Federal do Rio Grande do Sul, 91501-970, Porto Alegre, RS, Brazil

2 Departamento de Botânica, Universidade Federal do Rio Grande do Sul, 91501-970, Porto Alegre, RS, Brazil

3 Laboratório de Pesquisas em Interações Ecológicas, Universidade Federal do Pampa,97300-000, São Gabriel, RS, Brazil

* Corresponding author: suiane.bio@gmail.com 


\section{Introduction}

Plant-pollinator interactions play a fundamental role in biodiversity integrity (Potts et al. 2010) and are fundamental to plant population dynamics as they ensure population recruitment by fruit and seed set. In a plant community context, plant-pollinator interactions constitute one of the most important biotic factors, structuring community assemblage in temporal and spatial scales (Sargent \& Ackerly 2008). In addition to the functional importance of such interactions, they lead to a set of different pollination niches resulting from selective pressures played by distinct pollinators along the evolutionary history of plants (Johnson 2010).

Plant-pollinator interactions are under an increasing threat from human activity in grasslands (Corbet 2006; Weiner et al. 2014). The constant conversion of native landscapes could be putting a set of diverse organisms associated with still unknown ecological interactions at risk (Valiente-Banuet et al. 2015; French et al. 2017). Data about this mutualistic interaction from different plant communities and physiognomies could help us to understand the ecological-evolutionary processes that determine the occurrence patterns of a set of plant species (Wołowski et al. 2017) and to evaluate potential species extinction through plant-pollinator interactions (Memmott et al. 2007). Information about plant-pollinator interactions allied to reproductive system information can help in the proposition of optimal management strategies and conservation by the identification of pollen or pollinator limitation, specialised groups of plants (e.g., oil-producing flowers pollinated by specialised oil-collecting bees), vulnerable species in terms of reproductive outputs, and core species that can be used to attract a great richness of insects (Kearns et al. 1998). Additionally, the understanding of plant-pollinator interactions can provide information about the vulnerability of habitats, assessing the risks of local extinction of plants, animals, and the interactions among them (Simmons et al. 2020).

Although we have information about plant diversity and its heterogeneity along South Brazilian grasslands (Overbeck et al. 2007), there is a gap of studies concerning insect diversity in grasslands immersed in Atlantic Forest and Pampa phytogeographic domains (Bencke 2009) and their mutualistic plant interactions, mainly in Pampa (BPBES/REBIPP 2019). Most plant interaction studies in this region are associated with plant species of economic interest (Witter et al. 2014; Garibaldi et al. 2016; NunesSilva et al. 2016; BPBES/REBIPP 2019), besides some studies on bee foraging behaviour (taxonomy and plant source of pollen) (Schlindwein \& Wittmann 1995; Alves dos Santos 1997; Schlindwein 1998; Blochtein 2014). To understand the importance of pollinators as selective agents on floral traits, we must assess who they are and the level of specialisation or generalisation of these interactions in the community (Fenster et al. 2004). The degree of specialisation and generalisation of plant pollination systems could be an important tool to understand the ecology of pollinator services and aspects of reproductive isolation, speciation, extinction, and assembly of communities. The specialisation degree of the interactions also has several implications for community ecology and the resilience of pollinator services in the face of climate changes, land use, and all types of environmental disturbances (Armbruster 2017). In the past, most studies about plant-pollinator interactions in the Neotropics were conducted in forest, savanna-like, or Andean vegetation. However, the recent increase in the number of studies about plant-pollinator interactions from grassland vegetation could enable a better understanding of the pollination ecology of these landscapes (Freitas \& Sazima 2006)

The South Brazilian grasslands englobe two different phytogeographic domains (Coutinho 2006; Batalha 2011); the most southern portion belongs to the Pampa domain, while the northern portion is part of the Atlantic Rain Forest domain (hereafter, called ARF) (Overbeck et al. 2007). The Brazilian Pampa accounts for $63 \%$ of the Rio Grande do Sul State area, where grasslands with scattered shrubs and tree formations represent the dominant vegetation in the landscape (Carvalho et al. 2015). The Atlantic Rain Forest domain includes the grasslands of the Brazilian Plateau and is characterised by a mosaic of grasslands and forests in the northern half of the state of Rio Grande do Sul, Santa Catarina, and some areas of Paraná state. Including Pampa and ARF, an estimated 3,000 plant species exist in the South Brazilian grasslands (Boldrini 1997; Overbeck et al. 2007). Poaceae, Asteraceae, Cyperaceae, Fabaceae, Apiaceae, Oxalidaceae, Verbenaceae, and Iridaceae are the plant families with the highest richness in the Pampa (Overbeck et al. 2006; 2007; Andrade et al. 2019). Asteraceae, Apiaceae, and Verbenaceae are considered important floral reward sources to a wide range of pollinators (Pinheiro et al. 2008; Oleques et al. 2019). The unspecialised flowers of these plant groups and high population abundance, mainly Asteraceae, enhance the pollinator richness making these species crucial in pollination niche structuring in this region (Torres \& Galetto 2002; 2011).

In this paper, we provided a systematic review and a general perspective of plant-pollinator interactions in South Brazilian grasslands. Our goal was to describe the community-level interactions between plants and distinct pollinator groups of South Brazilian grasslands to understand the main pollination systems and level of specialisation of the flora region compared them to other South American grasslands. We also aimed to collaborate on a synthesis of knowledge and highlight the gaps and potentialities of plant-pollinator interactions studies in South Brazilian grasslands.

Our first expectation was that the high richness of unspecialised flowers, such as Asteraceae, would contribute 
to more generalised pollination systems with species being visited by two or more pollinator groups (Pinheiro et al. 2008; Oleques et. al. 2019). Regarding pollinator groups, our expectation was that bees would play an important role as pollinators of a great number of species, considering the diversity of bees in this region and the floral traits of the main plant families (Schlindwein 1998). Considering the richness of species from Asteraceae, Verbenaceae, Apiaceae and Iridaceae in South Brazilian Grasslands, our expectation about floral traits was that the majority of species would present open flowers (dish flowers), easily accessible by insects, with a short or absent floral tube (Herrera 1996).

\section{Materials and methods}

We compiled studies on plant-pollinator interactions with the help of extensive literature available on Google Scholar, ISI Web of Science, Scopus, Scielo, and the World Wide Web using the following search terms: "pollination AND Brazilian grasslands", "pollination AND South Brazil", "pollinators AND Southern Brazil, "flower visitors AND Southern Brazil, "pollination AND Pampa", "pollination AND South Brazilian Campos" (all terms were searched in both Portuguese and English). In addition, we searched for grassland data sets in the Interaction Web database (NCEAS) and used some information in unpublished manuscripts. We have selected papers with information about plantpollinator interactions or plant-floral-visitor interactions (with plant and animal taxonomic information) from Rio Grande do Sul, Santa Catarina and Paraná states without a temporal frame. We only considered studies based on field observations conducted in South Brazilian grasslands, which includes grassland vegetation of both Pampa and Atlantic Rain Forest domains. In our first search, we found five community-level papers. However, to avoid a bias in this review, we excluded two community papers: one of them the interactions of plant-pollinators were collected exclusively by the pollen loads of bees (Schlindwein 1998). The other was a study that covered both grassland and forest vegetation without differentiation in the results presentation (Mouga et al. 2012). Therefore, a total of 26 papers were reached and two were excluded by filters, resulting in 24 data sources (three studies at the community level and 21 case studies with one or a few plant species).

We categorised pollinators into eight distinct groups: bees, wasps, ants (Hymenoptera), flies (Diptera), beetles (Coleoptera), butterflies, hawkmoths (Lepidoptera), and hummingbirds (Apodiformes). Although species of ants are not usually considered pollinators, ants were treated as an independent category, herein, because they are frequently observed on flowers in many communities (García et al. 1996). To improve the discussion on bee-pollinated plants, we sub categorified bees as carpenter bees (Xylocopa and Ceratina), bumblebees (Bombus), oil-bees (Centris, Epicharis,
Arhyssoceble, Chaleopogenus, Lanthanomelissa), stingless bees (Plebeia, Mourella, Trigona, Melipona, Tetragonisca), and other bees, according to the subfamilies Colletinae, Megachiliinae, and Halictinae.

To classify the main pollination system of each plant family, we categorised specialists in a particular group using the species with more than $85 \%$ of visitors from that group. Species of plants with no group encompassing more than $85 \%$ of visitors were classified as insect/vertebrate generalists (Ollerton et al. 2006). Plant classification followed APG IV (2016) and Flora do Brasil 2020 (http://floradobrasil.jbrj. gov.br). We categorised floral type according to Faegri \& Van Der Pijl (1979), following the community level approach of Freitas \& Sazima (2006).

\section{Results}

\section{Plant-pollinator interactions studies}

Our search resulted in three plant community-level studies in the grasslands of the Pampa domain of Rio Grande do Sul (Pinheiro et al. 2008; Oleques et al. 2019; Beal-Neves et al. 2020). In addition, were found another 23 case pollination studies from grasslands of both phytogeographical domains, including one unpublished manuscript (RS Avila Jr unpubl. res.) and two MSc thesis (R. Becker; BC. Lopes) (Tab. 1). The studies consider to the systematic review were published from 2001 to 2020, being the most part of them published between 2017 and 2020 (see foot notes in Tab. 1). The latitudinal range varied from $31^{\circ} 48^{\prime} 36.96^{\prime \prime} \mathrm{S}, 52^{\circ} 24^{\prime} 53.13^{\prime \prime} \mathrm{W}$ (Pelotas, Rio Grande do Sul State) to $24^{\circ} 33^{\prime} 16.79^{\prime \prime}$ S, 50²13'58.26” W (Tibagí, Paraná State). This data set allowed us to collect information of 205 plant species from 125 genera and 40 families, approximately $16 \%$ of the flora from South Brazilian grasslands (Boldrini 1997) (Tab. 1, Fig. 1).

\section{Floral traits}

Most plant species recorded in this review presented a readily accessible dish flower type $(30.0 \%)$ and brush flowers (24.6\%). Narrow tube flowers were present in $15.7 \%$ of the species sampled. However, we found plant species presenting large tubes of approximately $10 \mathrm{~cm}$, belonging to Solanaceae Nicotiana alata Link \& Otto and Petunia spp. or in Oenothera affinis Cambess. Those with small and inconspicuous flowers were found in Anacardiaceae and Apiaceae species (Tab. 1, Fig. 2). Both nectar and pollen were the most common floral rewards observed in $53.6 \%$ of our sampled species. We recorded five Orchidaceae species $(3.3 \%)$ and one case of sexual mimicry with female flowers without floral rewards (Begonia cucullata Willd.). However, in the South Grasslands, $12.3 \%$ of the plant species offered just pollen as a floral reward, and pollen and oil were present in $7.3 \%$ of this set of plants. 
Table 1. Plant species, floral traits and potential pollinator groups of South Brazilian Grasslands with references.

\begin{tabular}{|c|c|c|c|}
\hline Families/ Species (reference) & Floral reward & Flower type & Pollinators Groups \\
\hline \multicolumn{4}{|l|}{ ACANTHACEAE } \\
\hline Ruellia hypericoides (Nees) Lindau ${ }^{(2)}$ & $\mathrm{n}, \mathrm{p}$ & tube & $\mathrm{B}(1), \mathrm{Bu}(2), \mathrm{Bt}(1), \mathrm{F}(1)$ \\
\hline \multicolumn{4}{|l|}{ AMARYLLIDACEAE } \\
\hline Nothoscordum gracile (Aiton) Stearn ${ }^{(1)}$ & $\mathrm{p}$ & dish & B (4), F (2) \\
\hline Nothoscordum montevidensis Beauverd (2), (23) & $\mathrm{p}$ & dish & $\mathrm{B}(1), \mathrm{Bt}(1), \mathrm{W}(1)$ \\
\hline Nothoscordum bonariense (Pers.) Beauverd (2) & $\mathrm{p}$ & dish & B (1), Bt (1),W (1), F (1) \\
\hline Zephyranthes sp. ${ }^{(1)}$ & $?$ & bell & B (1) \\
\hline Habranthus gracilifolius Herb. ${ }^{(3)}$ & $\mathrm{n}, \mathrm{p}$ & bell & $\mathrm{F}(10), \mathrm{Bt}(2), \mathrm{B}(4)$ \\
\hline Habranthus penduculosus Herb. ${ }^{(23)}$ & $?$ & bell & $\mathrm{F}(1)$ \\
\hline \multicolumn{4}{|l|}{ AMARANTHACEAE } \\
\hline Pfaffia tuberosa (Spreng.) Hicken ${ }^{(1),(2)}$ & $\mathrm{n}, \mathrm{p}$ & inconsp. & $\mathrm{B}(4), \mathrm{Bt}(4), \mathrm{Bu}(4), \mathrm{F}(8), \mathrm{W}$ (1) \\
\hline \multicolumn{4}{|l|}{ ANACARDIACEAE } \\
\hline Lithraea brasiliensis Marchand ${ }^{(1)}$ & $\mathrm{n}, \mathrm{p}$ & inconsp. & B (7), F (4), W (1) \\
\hline Schinus weinmanniaefolius Engl. (1) & $\mathrm{n}, \mathrm{p}$ & inconsp. & $\mathrm{B}(3), \mathrm{Bu}(1), \mathrm{W}(1)$ \\
\hline \multicolumn{4}{|l|}{ APIACEAE } \\
\hline Eryngium eriophorum Cham. \& Schltdl. ${ }^{(1)}$ & $\mathrm{n}, \mathrm{p}$ & inconsp. & $\mathrm{B}(10), \mathrm{Bu}(4), \mathrm{F}(7), \mathrm{W}(4)$ \\
\hline Eryngium horridum Malme ${ }^{(1),(2),(23)}$ & $\mathrm{n}, \mathrm{p}$ & inconsp. & $\mathrm{A}(3), \mathrm{B}(5), \mathrm{Bt}(13), \mathrm{Bu}(2), \mathrm{F}(13), \mathrm{W}$ (9) \\
\hline Eryngium megapotamicum Malme ${ }^{(1)}$ & $\mathrm{n}, \mathrm{p}$ & inconsp. & $\mathrm{B}(3), \mathrm{Bt}(1), \mathrm{Bu}(1), \mathrm{F}(3)$ \\
\hline Eryngium pristis Cham. \& Schltdl. (1) & $\mathrm{n}, \mathrm{p}$ & inconsp. & $\mathrm{B}(2), \mathrm{Bt}(3), \mathrm{F}(13), \mathrm{W}(4)$ \\
\hline Eryngium sanguisorba Cham. ${ }^{(1),(23)}$ & $\mathrm{n}, \mathrm{p}$ & inconsp. & $\mathrm{B}(6), \mathrm{Bt}(5) \mathrm{F}(15)$ \\
\hline Eryngium ciliatum Cham. \& Schltdl. (2),(23) & $\mathrm{n}, \mathrm{p}$ & inconsp. & $\mathrm{B}(8), \mathrm{Bt}(7), \mathrm{Bu}(2), \mathrm{F}(8)$ \\
\hline Eryngium elegans Cham. \& Schltdl. ${ }^{(23)}$ & $?$ & inconsp. & B (4), Bt (6), F (3) \\
\hline \multicolumn{4}{|l|}{ APOCYNACEAE } \\
\hline Blepharodon lineare (Decne.) Decne. ${ }^{(1)}$ & $\mathrm{n}, \mathrm{p}$ & dish & $\mathrm{W}(1)$ \\
\hline \multicolumn{4}{|l|}{ AQUIFOLIACEAE } \\
\hline Ilex dumosa Reissek ${ }^{(1)}$ & $\mathrm{n}, \mathrm{p}$ & dish & B (1), Bt (1), F (11), W (2) \\
\hline \multicolumn{4}{|l|}{ ARECACEAE } \\
\hline Butia capitata (Mart.) Becc. ${ }^{(1)}$ & $\mathrm{n}, \mathrm{p}$ & inconsp. & $\mathrm{B}(14), \mathrm{Bt}(1), \mathrm{F}(7), \mathrm{W}(5)$ \\
\hline \multicolumn{4}{|l|}{ ASTERACEAE } \\
\hline Achyrocline satureioides (Lam.) DC. (1), (2) & $\mathrm{n}, \mathrm{p}$ & tube (dish) & $\mathrm{A}(1), \mathrm{B}(3), \mathrm{F}(1), \mathrm{W}(5)$ \\
\hline Acmella bellidioides (Sm.) R.K. Jansen ${ }^{(1)}$ & $\mathrm{n}, \mathrm{p}$ & tube (dish) & $\mathrm{B}(1), \mathrm{F}(1)$ \\
\hline Acmella decumbens (Sm.) R.K. Jansen ${ }^{(1)}$ & $\mathrm{n}, \mathrm{p}$ & tube (dish) & $\mathrm{B}(7), \mathrm{F}(4)$ \\
\hline Aldama angustifolia (DC.) E.E.Schill. \& Panero ${ }^{(1)}$ & $\mathrm{n}, \mathrm{p}$ & tube (dish) & $\mathrm{B}(11), \mathrm{Bu}(5), \mathrm{Bt}(1), \mathrm{F}(2), \mathrm{W}(1)$ \\
\hline Aldama nudicaulis (Baker) E.E.Schill. \& Panero ${ }^{(1)}$ & $\mathrm{n}, \mathrm{p}$ & tube (dish) & B (1), F (1) \\
\hline Aspilia montevidensis (Spreng.) Kuntze ${ }^{(1),(2)}$ & $\mathrm{n}, \mathrm{p}$ & tube (dish) & $\mathrm{B}(9), \mathrm{Bu}(11), \mathrm{Bt}(6), \mathrm{F}(7), \mathrm{W}(2)$ \\
\hline Austroeupatorium laetevirens (Hook. \& Arn.) ${ }^{(1),(2),(23)}$ & $\mathrm{n}, \mathrm{p}$ & tube (brush) & $\mathrm{B}(1), \mathrm{Bt}(1), \mathrm{Bu}(3), \mathrm{F}(4)$ \\
\hline Austroeupatorium inulaefolium (Kunth) R.M.King \& H.Rob (23) & $\mathrm{n}, \mathrm{p}$ & tube (brush) & $\mathrm{Bt}(1)$ \\
\hline Baccharis articulata (Lam.) (2) & $\mathrm{n}, \mathrm{p}$ & tube (dish) & A (2), B (9), Bt (7), F (10), W (6) \\
\hline Baccharis crispa Spreng. ${ }^{(1),(2)}$ & $\mathrm{n}, \mathrm{p}$ & tube (brush) & $\mathrm{A}(3), \mathrm{B}(9), \mathrm{Bt}(2), \mathrm{F}(9), \mathrm{W}(6), \mathrm{Bu}(7)$ \\
\hline Baccharis cultrata Baker ${ }^{(1),(2)}$ & $\mathrm{n}, \mathrm{p}$ & tube (dish) & $\mathrm{B}(3), \mathrm{Bt}(1), \mathrm{F}(16), \mathrm{W}(8)$ \\
\hline Baccharis dracunculifolia DC. ${ }^{(1),(2)}$ & $\mathrm{n}, \mathrm{p}$ & tube (brush) & $\mathrm{A}(2), \mathrm{W}(1)$ \\
\hline Baccharis leucopappa DC. ${ }^{(23)}$ & $?$ & tube (brush) & Bt (1), F (2), W (3) \\
\hline Baccharis ochracea Spreng ${ }^{(1)}$ & $\mathrm{n}, \mathrm{p}$ & tube (brush) & $\mathrm{B}(1), \mathrm{Bu}(1), \mathrm{F}(3)$ \\
\hline Baccharis patens Baker ${ }^{(1)}$ & $\mathrm{n}, \mathrm{p}$ & tube (brush) & B (3), F (9), W (4) \\
\hline Baccharis pseudomyriocephala I.L. Teodoro ${ }^{(1)}$ & $\mathrm{n}, \mathrm{p}$ & tube (brush) & B (2), F (2), W (5) \\
\hline Baccharis psiadioides (Less.) Joch.Müll. (1) & $\mathrm{n}, \mathrm{p}$ & tube (dish) & B (2), F (1) \\
\hline Baccharis rufescens Spreng. ${ }^{(1)}$ & $\mathrm{n}, \mathrm{p}$ & tube (brush) & B (4), F (10), W (7) \\
\hline Baccharis riograndensis Malag. \& J.Vidal ${ }^{(23)}$ & $?$ & tube (brush) & W (1) \\
\hline Baccharis sessiliflora Vahl ${ }^{(1)}$ & $\mathrm{n}, \mathrm{p}$ & tube (brush) & B (2), F (6), W (6) \\
\hline Baccharis sagittalis (Less.) DC. ${ }^{(23)}$ & $?$ & tube (brush) & $\mathrm{F}(1), \mathrm{W}(2)$ \\
\hline Baccharis tridentata Vahl (1) & $\mathrm{n}, \mathrm{p}$ & tube (brush) & B (6), Bt (3), F (4), W (4) \\
\hline Barrosoa candolleana (Hook. \& Arn.) & $\mathrm{n}, \mathrm{p}$ & - & $\mathrm{W}(2)$ \\
\hline Calea uniflora Less ${ }^{(1),(23)}$ & $\mathrm{n}, \mathrm{p}$ & tube (dish) & $\mathrm{B}(4), \mathrm{Bt}(2), \mathrm{Bu}(1), \mathrm{F}(1)$ \\
\hline Campuloclinium macrocephalum ${ }^{(23)}$ & $?$ & & $\mathrm{~B}(1), \mathrm{Bt}(6)$ \\
\hline Chaptalia integerrima (Vell.) Burk. (1), (2) & $\mathrm{n}, \mathrm{p}$ & tube (brush) & B (1) \\
\hline Chrysolaena flexuosa (Sims) H.Rob. (1), (2), (23) & $\mathrm{n}, \mathrm{p}$ & tube (brush) & $\mathrm{B}(8), \mathrm{Bu}(5), \mathrm{Bt}(6), \mathrm{F}(3), \mathrm{W}(1)$ \\
\hline
\end{tabular}


Table 1. Cont.

\begin{tabular}{|c|c|c|c|}
\hline Families/ Species (reference) & Floral reward & Flower type & Pollinators Groups \\
\hline Chromolaena ascendens (Sch.Bip. Ex Baker) R.M.King \& H. Rob. ${ }^{(23)}$ & $?$ & tube (brush) & $\mathrm{B}(1), \mathrm{Bt}(3)$ \\
\hline Chromolaena hirsuta (Hook. \& Arn.) R.M.King \& H.Rob. ${ }^{(23)}$ & ? & tube (brush) & $\mathrm{Bt}(1)$ \\
\hline Chromolaena laevigata (Lam.) R.M.King \& H.Rob. ${ }^{(23)}$ & $?$ & tube (brush) & $\mathrm{Bt}(1)$ \\
\hline Campuloclinium macrocephalum (Less) DC. ${ }^{(23)}$ & ? & & $\mathrm{B}(1) \mathrm{Bt}(4)$ \\
\hline Dasyphyllum brasiliense (Spreng.) Cabrera ${ }^{(4)^{*}}$ & $\mathrm{n}, \mathrm{p}$ & tube (brush) & $\mathrm{B}(2), \mathrm{Bu}(2), \mathrm{F}(2), \mathrm{W}(1)$ \\
\hline Disynaphia ligulifolia (Hook. \& Arn.) R.M.King \& H.Rob. ${ }^{(23)}$ & ? & & $\mathrm{B}(3), \mathrm{Bt}(2), \mathrm{F}(2)$ \\
\hline Eupatorium ligulaefolium Hook. \& Arn. ${ }^{(1),(2)}$ & $\mathrm{n}, \mathrm{p}$ & tube (brush) & $\mathrm{B}(1), \mathrm{Bu}(1), \mathrm{F}(1)$ \\
\hline Eupatorium serratum Spreng. ${ }^{(1),(2)}$ & $\mathrm{n}, \mathrm{p}$ & tube (brush) & $\mathrm{B}(3), \mathrm{Bu}(1), \mathrm{Bt}(1), \mathrm{F}(5), \mathrm{W}(1)$ \\
\hline Eupatorium subhastatum Hook. \& Arn. . (1), (2) & $\mathrm{n}, \mathrm{p}$ & tube (brush) & $\mathrm{B}(1)$ \\
\hline Hieracium commersonii Monnier ${ }^{(1),(2),(23)}$ & $\mathrm{n}, \mathrm{p}$ & tube (dish) & $\mathrm{B}(3), \mathrm{F}(1)$ \\
\hline Grazielia intermedia (DC.) R.M.King \& H.Rob. ${ }^{(23)}$ & ? & tube (brush) & $\mathrm{B}(2), \mathrm{Bt}(5), \mathrm{Bu}(1), \mathrm{F}(2)$ \\
\hline Gyptis pinnatifida Cass. ex R.M.King \& H.Rob. ${ }^{(23)}$ & ? & tube (brush) & $\mathrm{Bu}(2), \mathrm{F}(1)$ \\
\hline Stenocephalum megapotamicum (Spreng.) Sch.Bip. ${ }^{(23)}$ & ? & tube (brush) & $\mathrm{B}(2)$ \\
\hline Holocheilus brasiliensis (L.) Cabrera $^{(1),(2)}$ & $\mathrm{n}, \mathrm{p}$ & tube (dish) & $\mathrm{B}(3), \mathrm{Bt}(1), \mathrm{F}(1)$ \\
\hline Hypochaeris megapotamica Cabr. ${ }^{(1),(2)}$ & $\mathrm{n}, \mathrm{p}$ & tube (brush) & $\mathrm{B}(1)$ \\
\hline Hypochaeris variegata (Lam.) Baker ${ }^{(1),(2)}$ & $\mathrm{n}, \mathrm{p}$ & tube (brush) & $\mathrm{B}(2), \mathrm{F}(1)$ \\
\hline Lessingianthus polyphyllus (Sch.Bip. ex Baker) H.Rob. ${ }^{(1),(2),(23)}$ & $\mathrm{n}, \mathrm{p}$ & tube (brush) & $\mathrm{B}(4), \mathrm{Bt}(1), \mathrm{Bu}(1), \mathrm{F}(1)$ \\
\hline Pamphalea commersonii Cass. & $?$ & & $\mathrm{~F}(1)$ \\
\hline Porophyllum laceolatum DC. ${ }^{(1),(2)}$ & $\mathrm{n}, \mathrm{p}$ & tube (brush) & B (4), W (2) \\
\hline Porophyllum curticeps Malme ${ }^{(23)}$ & $?$ & tube (brush) & $\mathrm{B}(1), \mathrm{Bt}(3)$ \\
\hline Pterocaulon alopecuroides (Lam.) DC. ${ }^{(1),(2)}$ & $\mathrm{n}, \mathrm{p}$ & tube (brush) & $\mathrm{W}(1)$ \\
\hline Pterocaulon angustifolium DC. ${ }^{(23)}$ & $?$ & tube (brush) & $\mathrm{B}(1), \mathrm{Bt}(2), \mathrm{W}(3)$ \\
\hline Schlechtendalia luzulifolia Less. ${ }^{(1),(2),(23)}$ & $\mathrm{n}, \mathrm{p}$ & tube (brush) & $\mathrm{B}(7), \mathrm{Bt}(1)$ \\
\hline Senecio heterotrichius DC. ${ }^{(2)}$ & $\mathrm{n}, \mathrm{p}$ & tube (dish) & $\mathrm{B}(3), \mathrm{Bu}(5), \mathrm{Bt}(3), \mathrm{W}(3)$ \\
\hline Senecio leptolobus DC. ${ }^{(1),(2)}$ & $\mathrm{n}, \mathrm{p}$ & tube (dish) & $\mathrm{B}(5), \mathrm{Bu}(2), \mathrm{F}(4), \mathrm{W}(3)$ \\
\hline Senecio madagascariensis Poir. ${ }^{(2)}$ & $\mathrm{n}, \mathrm{p}$ & tube (dish) & $\mathrm{B}(6), \mathrm{Bu}(5), \mathrm{Bt}(3), \mathrm{F}(5)$ \\
\hline Stenachaenium megapotamicum (Spreng.) Baker ${ }^{(2)}$ & $\mathrm{n}, \mathrm{p}$ & tube (brush) & $\mathrm{Bu}(1)$ \\
\hline Stevia cinerascens Sch. Bip. ex Baker ${ }^{(1)}$ & $\mathrm{n}, \mathrm{p}$ & tube (dish) & B (4) \\
\hline Symphyopappus cuneatus (DC.) Sch.Bip. ex Baker ${ }^{(1)}$ & $\mathrm{n}, \mathrm{p}$ & tube (brush) & $\mathrm{B}(5)$ \\
\hline Symphyopappus reticulatus Baker (23) & $?$ & tube (brush) & $\mathrm{B}(1)$ \\
\hline Solidago chilensis Meyen ${ }^{(23)}$ & ? & & $\mathrm{W}(1)$ \\
\hline Verbesina subdiscoidea Toledo ${ }^{(1)}$ & $\mathrm{n}, \mathrm{p}$ & tube (dish) & B (4), W (2) \\
\hline Verbesina sordescens ${ }^{(23)}$ & $?$ & tube (dish) & $\mathrm{B}(1)$ \\
\hline Vernonanthura montevidensis (Spreng.) H. Rob. ${ }^{(1)}$ & $\mathrm{n}, \mathrm{p}$ & tube (brush) & $\mathrm{B}(1), \mathrm{F}(3)$ \\
\hline Vernonanthura nudiflora (Less.) H.Rob. (1), (2), (23) & $\mathrm{n}, \mathrm{p}$ & tube (brush) & $\mathrm{B}(4), \mathrm{Bu}(8), \mathrm{Bt}(4), \mathrm{F}(4)$ \\
\hline Vernonia hypochaeris Schreb. ${ }^{(23)}$ & $?$ & tube (brush) & $\mathrm{B}(1), \mathrm{Bt}(1)$ \\
\hline Verbesina sordescens DC. ${ }^{(23)}$ & ? & tube (dish) & $\mathrm{B}(1)$ \\
\hline Trixis nobilis (Vell.) Katinas ${ }^{(23)}$ & ? & tube (brush) & $\mathrm{Bt}(1)$ \\
\hline \multicolumn{4}{|l|}{ BEGONIACEAE } \\
\hline Begonia cucullata Willd. ${ }^{(1),(5)}$ & $\mathrm{p}$ & dish & $\mathrm{B}(4), \mathrm{F}(1)$ \\
\hline \multicolumn{4}{|l|}{ BORAGINACEAE } \\
\hline Cordia verbenacea DC.(1) & $\mathrm{n}, \mathrm{p}$ & bell & $\mathrm{B}(3), \mathrm{Bu}(1), \mathrm{F}(1), \mathrm{W}$ (3) \\
\hline Varronia curassavica Jacq. ${ }^{(23)}$ & $?$ & bell & $\mathrm{B}(1), \mathrm{Bt}(2), \mathrm{F}(1), \mathrm{W}(1)$ \\
\hline \multicolumn{4}{|l|}{ BROMELIACEAE } \\
\hline Dyckia maritima Baker ${ }^{(1)}$ & $\mathrm{n}, \mathrm{p}$ & tube & $\mathrm{B}(3)$ \\
\hline Dyckia leptostachya Baker ${ }^{(23)}$ & $?$ & tube & $\mathrm{B}(1)$ \\
\hline Dyckia choristaminea Baker ${ }^{(23)}$ & ? & tube & $\mathrm{F}(1)$ \\
\hline \multicolumn{4}{|l|}{ CACTACEAE } \\
\hline Opuntia monacantha Haw. ${ }^{(1)}$ & $\mathrm{p}$ & dish & B (4) \\
\hline Parodia crassigiba (Ritter) N.P. Taylor ${ }^{(6)}$ & $\mathrm{p}$ & dish & $\mathrm{B}(7)$ \\
\hline $\begin{array}{c}\text { Parodia neohorstii (S.Theun.) } \\
\text { N.P. Taylor }{ }^{(7)}\end{array}$ & $\mathrm{p}$ & dish & $\mathrm{B}(7)$ \\
\hline Parodia ottonis (Lehm.) N. P. Taylor ${ }^{(23)}$ & $\mathrm{p}$ & dish & $\mathrm{B}(2), \mathrm{Bt}(1)$ \\
\hline Cereus hildmannianus K.Schum. ${ }^{(26)}$ & $\mathrm{n}, \mathrm{p}$ & tube & $\mathrm{Ha}(3)$ \\
\hline \multicolumn{4}{|l|}{ CAMPANULACEAE } \\
\hline Wahlenbergia linarioides (Lam.) A.DC. (2), (23) & $\mathrm{n}, \mathrm{p}$ & bell & $\mathrm{B}(1), \mathrm{F}(1)$ \\
\hline
\end{tabular}


Table 1. Cont.

\begin{tabular}{|c|c|c|c|}
\hline Families/Species (reference) & Floral reward & Flower type & Pollinators Groups \\
\hline \multicolumn{4}{|l|}{ CAPRIFOLIACEAE } \\
\hline Valeriana chamaedryfolia Cham. \& Schltdl. ${ }^{(1)}$ & $\mathrm{n}, \mathrm{p}$ & inconsp. & B (4), W (7) \\
\hline \multicolumn{4}{|l|}{ CELASTRACEAE } \\
\hline Maytenus cassineformis Reissek ${ }^{(1)}$ & $\mathrm{n}, \mathrm{p}$ & inconsp. & $\mathrm{F}(3), \mathrm{W}(1)$ \\
\hline \multicolumn{4}{|l|}{ COMMELINACEAE } \\
\hline Commelina sp. ${ }^{(1)}$ & $\mathrm{p}$ & dish & $\mathrm{B}(1), \mathrm{F}(1)$ \\
\hline Tradescantia sp. $^{(1)}$ & $\mathrm{p}$ & dish & B (2) \\
\hline Commelina erecta L. ${ }^{(23)}$ & $\mathrm{p}$ & dish & $\mathrm{B}(1), \mathrm{Bt}(1)$ \\
\hline \multicolumn{4}{|l|}{ CONVOLVULACEAE } \\
\hline Evolvulus glomeratus Ness \& Mart. ${ }^{(2),(23)}$ & $\mathrm{n}, \mathrm{p}$ & dish & B (16), F (3) \\
\hline Evolvulus sericeus Sw. ${ }^{(2)}$ & $\mathrm{n}, \mathrm{p}$ & dish & $\mathrm{B}(1), \mathrm{F}(2)$ \\
\hline Ipomea sp. ${ }^{(23)}$ & $?$ & bell & $\mathrm{B}(1)$ \\
\hline Ipomea uruguayensis Meisn. ${ }^{(23)}$ & $?$ & bell & $\mathrm{B}(1), \mathrm{Bt}(2)$ \\
\hline \multicolumn{4}{|l|}{ ERICACEAE } \\
\hline Agarista eucalyptoides (Cham. \& Schltdl.) G.Don ${ }^{(1)}$ & $\mathrm{n}, \mathrm{p}$ & tube & B (1) \\
\hline \multicolumn{4}{|l|}{ EUPHORBIACEAE } \\
\hline Croton gnaphali Baill(2) & $\mathrm{n}, \mathrm{p}$ & dish & B (13), F (9), W (8) \\
\hline Euphorbia selloi (Klotzsch \& Garcke) Boiss. ${ }^{(2)}$ & $\mathrm{n}, \mathrm{p}$ & dish & B (4), F (2), W (2) \\
\hline \multicolumn{4}{|l|}{ FABACEAE } \\
\hline Aeschynomene falcata (Poir.) DC. ${ }^{(2)}$ & $\mathrm{n}, \mathrm{p}$ & flag & $\mathrm{Bu}(1)$ \\
\hline Centrosema virginianum (L.) Benth. ${ }^{(23)}$ & $?$ & $?$ & $\mathrm{~B}(1)$ \\
\hline Collaea stenophylla (Hook. \& Arn.) Benth. ${ }^{(23)}$ & $?$ & flag & $\mathrm{B}(1), \mathrm{Bt}(5), \mathrm{F}(2)$ \\
\hline Chamaecrista repens (Vogel) H.S. Irwin \& Barneby. ${ }^{(23)}$ & $?$ & flag & $\mathrm{B}(3), \mathrm{Bt}(1)$ \\
\hline Crotalaria twediana Benth. ${ }^{(2)}$ & $\mathrm{n}, \mathrm{p}$ & flag & B (2) \\
\hline Erythrina crista-galli L. ${ }^{(8)}$ & $\mathrm{n}, \mathrm{p}$ & flag & $\mathrm{B}(2), \mathrm{Hu}(1)$ \\
\hline Desmodium cuneatum Hook. \& Arn. ${ }^{(1)}$ & $\mathrm{n}, \mathrm{p}$ & flag & B (1), W (1) \\
\hline Desmodium incanum DC. (2) & $\mathrm{n}, \mathrm{p}$ & flag & B (2), F (1) \\
\hline Macroptilium prostatum (Benth.) Urb. ${ }^{(2)}$ & $\mathrm{n}, \mathrm{p}$ & flag & B (1), W (3) \\
\hline Mimosa sanguinolenta Barneby ${ }^{(23)}$ & $?$ & brush & B (1) \\
\hline Mimosa dolens (Benth.) Barneby ${ }^{(23)}$ & $?$ & brush & $\mathrm{B}(1), \mathrm{Bt}(3), \mathrm{F}(1)$ \\
\hline Mimosa schleidenii Herter ${ }^{(1)}$ & $\mathrm{n}, \mathrm{p}$ & brush & B (3) \\
\hline Prosopis affinis Spreng. ${ }^{(9)}$ & $?$ & brush & $\mathrm{B}(?)$ \\
\hline Stylosanthes leiocarpa Vogel (2) & $?$ & flag & $\mathrm{B}(2), \mathrm{Bt}(1), \mathrm{F}(1)$ \\
\hline Vachellia caven (Molina) Seigler \& Ebinger ${ }^{(9)}$ & $\mathrm{p}$ & brush & $\mathrm{B}(?), \mathrm{W}(?)$ \\
\hline Zornia sp. (2) & $?$ & flag & $\mathrm{W}(1)$ \\
\hline \multicolumn{4}{|l|}{ LOASACEAE } \\
\hline Blumenbachia amana T.Henning \& Weigend ${ }^{(19)}$ & $\mathrm{n}, \mathrm{p}$ & dish & B (1) \\
\hline Blumenbachia insignis Schrad. ${ }^{(20)}$ & $\mathrm{n}, \mathrm{p}$ & dish & B (1) \\
\hline \multicolumn{4}{|l|}{ GESNERIACEAE } \\
\hline Sinningia allagophylla (Mart.) Wiehler ${ }^{(1)}$ & $\mathrm{n}, \mathrm{p}$ & tube & B (5) \\
\hline \multicolumn{4}{|l|}{ HYPERICACEAE } \\
\hline Hypericum brasiliense Choisy ${ }^{(1)}$ & $\mathrm{p}$ & dish & B (1), Bt (1) \\
\hline \multicolumn{4}{|l|}{ IRIDACEAE } \\
\hline Calydorea alba Roitman \& J.A.Castillo ${ }^{(21)}$ & $\mathrm{p}$ & dish & Pollen-B (3) \\
\hline Cipura paludosa Aubl. (21) & $\mathrm{p}$ & dish & Pollen-B (1) \\
\hline Cypella amplimaculata Chauveau \& L.Eggers ${ }^{(21)}$ & $o, p$ & dish & Oil-B (1),Pollen-B (3) \\
\hline Cypella herbertii Hook ${ }^{(22),(21)}$ & $o, p$ & dish & Oil-B (3), Pollen-B (3) \\
\hline Cypella pusilla (Link \& Otto) Benth. ${ }^{(21)}$ & $o, p$ & dish & Oil-B (2), Pollen-B (1) \\
\hline Herbertia lahue (Molina) Goldblatt ${ }^{(21)}$ & $o, p$ & dish & Oil-B (3), Pollen-B (8) \\
\hline Herbertia pulchella Sweet ${ }^{(16),(1),(21)}$ & $o, p$ & dish & Oil-B (3),Pollen-B (15) \\
\hline Kelissa brasiliensis (Baker) Ravenna ${ }^{(21)}$ & $o, p$ & dish & Oil-B (1),Pollen-B (6) \\
\hline Sisyrinchium macrocephalum Graham ${ }^{(1)}$ & $o, p$ & dish & Pollen-B (3) \\
\hline Sisyrinchium micranthum Cav. ${ }^{(17),(1),(2)}$ & $o, p$ & tube & Oil-B (4), Pollen-B (3) \\
\hline Sisyrinchium osteniamum Beauv ${ }^{(1)}$ & $o, p$ & $?$ & Pollen-B (1) \\
\hline Sisyrinchium scariosum I.M. ${ }^{(1)}$ & $o, p$ & dish & Oil-B (1), Pollen-B (2) \\
\hline Sisyrinchium sellowianum Klatt ${ }^{(1)}$ & $o, p$ & dish & Oil-B (1), Pollen-B (2) \\
\hline
\end{tabular}


Table 1. Cont.

\begin{tabular}{|c|c|c|c|}
\hline Families/Species (reference) & Floral reward & Flower type & Pollinators Groups \\
\hline Sisyrinchium palmifolium L. ${ }^{(1)}$ & $\mathrm{o}, \mathrm{p}$ & dish & Pollen-B (1) \\
\hline \multicolumn{4}{|l|}{ LAMIACEAE } \\
\hline Glechon marifolia Benth. $^{(1)}$ & $\mathrm{n}, \mathrm{p}$ & gullet & B (9), W (5) \\
\hline Peltodon longipes Kunth. ex Benth. ${ }^{(2)}$ & $?$ & flag & $\mathrm{B}(3), \mathrm{Bu}(2), \mathrm{A}(1)$ \\
\hline Glechon ciliata Benth. ${ }^{(23)}$ & $?$ & gullet & $\mathrm{Bt}(1)$ \\
\hline \multicolumn{4}{|l|}{ MALVACEAE } \\
\hline Abutilon malachroides A.St.-Hil. \& Naudin & $?$ & & $\mathrm{~B}(1), \mathrm{Bt}(1)$ \\
\hline Wissadula glechomifolia Hassl. & $?$ & & B (1) \\
\hline Waltheria douradinha A. St.-Hil. (2), (23) & $\mathrm{n}, \mathrm{p}$ & dish & $\mathrm{B}(2), \mathrm{Bt}(4), \mathrm{W}(1)$ \\
\hline Pavonia friesii Krapov. ${ }^{(23)}$ & $?$ & dish & $\mathrm{F}(2)$ \\
\hline \multicolumn{4}{|l|}{ MELASTOMATACEAE } \\
\hline Tibouchina gracilis (Bonpl.) Cogn. ${ }^{(1),(2),(23)}$ & $\mathrm{p}$ & dish & $\mathrm{B}(1), \mathrm{Bt}(1), \mathrm{Bu}(2)$ \\
\hline Tibouchina hatschbachii Wurdack ${ }^{(25) *}$ & $\mathrm{p}$ & dish & $\mathrm{B}(3)$ \\
\hline \multicolumn{4}{|l|}{ MYRTACEAE } \\
\hline Blepharocalyx salicifolius (Kunth) O.Berg ${ }^{(1)}$ & $\mathrm{p}$ & brush & $\mathrm{Bt}(1)$ \\
\hline Campomanesia aurea O.Berg ${ }^{(1),(23)}$ & $\mathrm{p}$ & brush & $\mathrm{B}(1), \mathrm{Bt}(2), \mathrm{F}(3)$ \\
\hline Myrcia palustris DC. ${ }^{(1)}$ & $\mathrm{p}$ & brush & $\mathrm{B}(1), \mathrm{Bu}(1), \mathrm{F}(2)$ \\
\hline Myrciaria cuspidata O.Berg ${ }^{(1)}$ & $\mathrm{n}, \mathrm{p}$ & brush & $\mathrm{B}(2), \mathrm{Bt}(1), \mathrm{F}(4)$ \\
\hline Pisidum cattleyanum Sabine ${ }^{(1)}$ & $\mathrm{n}, \mathrm{p}$ & brush & $\mathrm{Bt}(2)$ \\
\hline \multicolumn{4}{|l|}{ ORCHIDACEAE } \\
\hline Epidendrum fulgens Brongn. ${ }^{(1)}$ & $\mathrm{n}$ & gullet & $\mathrm{Bu}(1)$ \\
\hline Cattleya intermedia Graham ex Hook. ${ }^{(10) *}$ & none & gullet & B (4) \\
\hline Cattleya tigrina A.Rich. ${ }^{(10)^{*}}$ & none & gullet & B (1) \\
\hline Cattleya purpurata Lindl. ${ }^{(10)^{*}}$ & none & gullet & B (2) \\
\hline Cattleya coccinea Lindl. ${ }^{(10)^{*}}$ & none & gullet & $\mathrm{Hu}(1)$ \\
\hline Chloraea membranacea Lindl. ${ }^{(11)^{*}}$ & none & gullet & B (3) \\
\hline Habenaria megapotamensis Hoehne ${ }^{(12) *}$ & $\mathrm{n}$ & tube & $\mathrm{Ha}(1)$ \\
\hline Habenaria johannensis Barb. Rodr. ${ }^{(12)^{*}}$ & $\mathrm{n}$ & tube & $\mathrm{Ha}(2)$ \\
\hline Habenaria macronectar (Vell.) Hoehne ${ }^{(12)}{ }^{*}$ & $\mathrm{n}$ & tube & $\mathrm{Ha}(1)$ \\
\hline Habenaria montevidensis Spreng. ${ }^{(12)}{ }^{*}$ & $\mathrm{n}$ & tube & $\mathrm{Bu}(4)$ \\
\hline \multicolumn{4}{|l|}{ OXALIDACEAE } \\
\hline Oxalis eriocarpa DC. (2) & $\mathrm{n}, \mathrm{p}$ & bell & $\mathrm{B}(1), \mathrm{Bt}(3), \mathrm{Bu}(1)$ \\
\hline Oxalis conorrhiza Jacq. ${ }^{(2)}$ & $\mathrm{n}, \mathrm{p}$ & bell & $\mathrm{Bt}(1)$ \\
\hline Oxalis lasiopetala Zucc. ${ }^{(2)}$ & $\mathrm{n}, \mathrm{p}$ & bell & $\mathrm{Bu}(4), \mathrm{F}(3)$ \\
\hline \multicolumn{4}{|l|}{ OROBANCHACEAE } \\
\hline Buchnera longifolia Kunth ${ }^{(2)}$ & $?$ & tube & $\mathrm{B}(1), \mathrm{Bu}(1)$ \\
\hline \multicolumn{4}{|l|}{ PASSIFLORACEAE } \\
\hline Piriqueta suborbicularis (A.St.-Hil. \& Naudin) Arbo ${ }^{(1)}$ & $\mathrm{n}, \mathrm{p}$ & dish & B (1) \\
\hline \multicolumn{4}{|l|}{ PORTULACACEAE } \\
\hline Portulaca hirsutissima Cambess. ${ }^{(18)}$ & $\mathrm{p}$ & dish & $\mathrm{B}(5)$ \\
\hline Portulaca grandiflora Hook. ${ }^{(18)}$ & $\mathrm{p}$ & dish & B (5) \\
\hline \multicolumn{4}{|l|}{ PLANTAGINACEAE } \\
\hline Mecardonia tenella (Cham. \& Schltdl.) Pennell (1),(2) & $o, p$ & gullet & Pollen-B (4), Bt (1) \\
\hline Mecardonia procumbens (Mill.) Small (1), (2) & $o, p$ & gullet & Pollen-B (2) \\
\hline Scoparia dulcis L. (1), (2) & $\mathrm{p}$ & dish & $\mathrm{B}(2), \mathrm{F}(1)$ \\
\hline Angelonia integerrima Spreng. (1), (2), (24) & $o, p$ & bell & Oil-B (7), Pollen-B (9), F (2), W (2) \\
\hline \multicolumn{4}{|l|}{ POLYGALACEAE } \\
\hline Monnina oblongifolia Arechav. ${ }^{(1)}$ & $\mathrm{p}, \mathrm{n}$ & flag & $\mathrm{B}(10)$ \\
\hline Polygala pulchella A. St.-Hil. (1) & $\mathrm{p}, \mathrm{n}$ & flag & $\mathrm{Bt}(2), \mathrm{Bu}(2), \mathrm{F}(1)$ \\
\hline \multicolumn{4}{|l|}{ RUBIACEAE } \\
\hline Borreria brachystemonoides Cham. \& Schltdl. ${ }^{(1),(2)}$ & $\mathrm{n}, \mathrm{p}$ & tube & $\mathrm{B}(2), \mathrm{F}(2)$ \\
\hline Borreria capitata (Ruiz \&Pav.) DC. (1), (2) & $\mathrm{n}, \mathrm{p}$ & tube & $\mathrm{B}(1), \mathrm{Bu}(2), \mathrm{F}(2)$ \\
\hline Borreria verticillata (L.) G.Mey. ${ }^{(1),(2)}$ & $\mathrm{n}, \mathrm{p}$ & tube & $\mathrm{B}(1), \mathrm{Bu}(1), \mathrm{F}(3), \mathrm{W}(1)$ \\
\hline Borreria eryngioides Cham. \& Schltdl. (1), (2) & $\mathrm{n}, \mathrm{p}$ & tube & B (3), F (1), W (1) \\
\hline Richardia grandiflora (Cham. \& Schltdl.) Steud. (1),(2) & $\mathrm{n}, \mathrm{p}$ & tube & $\mathrm{B}(9), \mathrm{Bu}(2), \mathrm{Bt}(3), \mathrm{F}(3), \mathrm{W}$ (1) \\
\hline Galianthe fastigiata Griseb. ${ }^{(1),(2)}$ & $\mathrm{n}, \mathrm{p}$ & tube & $\mathrm{B}(16), \mathrm{Bu}(5), \mathrm{F}(3), \mathrm{W}(8)$ \\
\hline
\end{tabular}


Table 1. Cont.

\begin{tabular}{|c|c|c|c|}
\hline Families/Species (reference) & Floral reward & Flower type & Pollinators Groups \\
\hline Chiococca alba (L.) Hitchc ${ }^{(1),(2)}$ & $\mathrm{n}, \mathrm{p}$ & bell & $\mathrm{B}(1)$ \\
\hline Faramea martiana Müll.Arg. ${ }^{(1),(2)}$ & $\mathrm{n}, \mathrm{p}$ & tube & $\mathrm{B}(7), \mathrm{F}(1), \mathrm{W}(6)$ \\
\hline \multicolumn{4}{|l|}{ SOLANACEAE } \\
\hline Calibrachoa heterophylla (Sendtn.) Wijsman ${ }^{(14)}$ & $\mathrm{n}, \mathrm{p}$ & tube & $\mathrm{B}(1)$ \\
\hline Petunia axillaris (Lam.) Britton et al. ${ }^{(13)}$ & $\mathrm{n}, \mathrm{p}$ & tube & $\mathrm{Ha}(?)$ \\
\hline Petunia exserta J.R. Stehm. ${ }^{(14)}$ & $\mathrm{n}, \mathrm{p}$ & tube & $\mathrm{Hu}$ \\
\hline Petunia integrifolia (Hook.) Schinz \& Thell. .13) $^{(13)}$ & $\mathrm{n}, \mathrm{p}$ & tube & $\mathrm{B}(1)$ \\
\hline Petunia secreta Stehmann \& Semir ${ }^{(14)}$ & $\mathrm{n}, \mathrm{p}$ & tube & $\mathrm{B}(4)$ \\
\hline Nicotiana alata Link \& Otto ${ }^{(15)}$ & $\mathrm{n}, \mathrm{p}$ & tube & $\mathrm{Ha}(2)$ \\
\hline Nicotiana forgetiana Hemsl. ${ }^{(15)}$ & $\mathrm{n}, \mathrm{p}$ & tube & $\mathrm{Hu}(1)$ \\
\hline Solanum sisymbriifolium Lam. ${ }^{(1)}$ & $\mathrm{p}$ & dish & $\mathrm{B}(1)$ \\
\hline \multicolumn{4}{|l|}{ STYRACACEAE } \\
\hline Styrax leprosus Hook. \& Arn. ${ }^{(1)}$ & $\mathrm{p}$ & dish & $\mathrm{B}(7)$ \\
\hline \multicolumn{4}{|l|}{ VERBENACEAE } \\
\hline Lantana camara L. (1), (2), (23) & $\mathrm{n}, \mathrm{p}$ & tube & $\mathrm{B}(1), \mathrm{Bu}(2)$ \\
\hline Lippia angustifolia Cham. (1), (2) & $\mathrm{n}, \mathrm{p}$ & tube & $\mathrm{B}(4), \mathrm{F}(2), \mathrm{W}(1)$ \\
\hline Stachytarpheta cayennensis (Rich.) Vahl ${ }^{(1),(2)}$ & $\mathrm{n}, \mathrm{p}$ & tube & $\mathrm{B}(4), \mathrm{Bu}(15), \mathrm{F}(2)$ \\
\hline Verbena sagittalis Cham. (1), (2) & $\mathrm{n}, \mathrm{p}$ & tube & $\mathrm{B}(2), \mathrm{W}(1)$ \\
\hline Verbena litoralis Kunth ${ }^{(1),(2)}$ & $\mathrm{n}, \mathrm{p}$ & tube & $\mathrm{B}(3), \mathrm{Bu}(12), \mathrm{F}(2), \mathrm{W}(3)$ \\
\hline Lippia turnerifolia Cham. ${ }^{(1),(2)}$ & $\mathrm{n}, \mathrm{p}$ & tube & $\mathrm{B}(2), \mathrm{Bu}(1), \mathrm{F}(2)$ \\
\hline Verbena ephedroides Cham. ${ }^{(1),(2)}$ & $\mathrm{n}, \mathrm{p}$ & tube & $\mathrm{F}(3)$ \\
\hline \multicolumn{4}{|l|}{ VIOLACEAE } \\
\hline Hybanthus bicolor (Saint-Hilaire) Baill. ${ }^{(2)}$ & $\mathrm{n}, \mathrm{p}$ & flag & $\mathrm{B}(1), \mathrm{Bu}(1)$ \\
\hline
\end{tabular}

$\mathrm{n}=$ nectar, $\mathrm{p}=$ pollen, $\mathrm{o}=\mathrm{oil}, \mathrm{?}=$ data not available, ${ }^{*}$ studies conducted in ARFB (Atlantic Rain Forest Biome); A = ants, B = bee, $\mathrm{Bt}=$ beetles, $\mathrm{Bu}=$ butterflies, $\mathrm{F}=$ flies, $\mathrm{Ha}=$ hawkmoths, $\mathrm{Hu}=$ hummingbrids, $\mathrm{W}=$ wasps; (1) Pinheiro et al. 2008; (2) Oleques et al. 2019; (3) Streher et al. 2018; (4) Lopes 2017; (5) Avila Jr et al. 2017; (6) R. Avila Jr (personal communication); (7) Cerceau et al. 2019; (8) Costa \& Morais 2008; (9) Lôbo \& Stefennon 2018; (10) Caballero-Villalobos et al. 2017; (11) Sanguinetti et al. 2012 ; (12) Pedron et al. 2012; (13) Ando et al. 2001; (14) Rodrigues et al. 2018; (15) Ippolito et al. 2004; (16) Schlindwein 1998;(17) Schlindwein \& Martins 2000; (18) Pinto \& Schlindwein 2014; (19) Siriani-Oliveira et al. 2018; (20) Siriani-Oliveira et al. 2019; (21) Oleques et al. 2020; (22) Schlindwein \& Martins 2000; (23) Beal-Neves et al. 2020; (24) Martins et al. 2013; (25) Maia et al. 2018; (26) Becker 2020.

\section{Pollination systems}

Among all plant species, $56.7 \%$ were visited by more than one group of pollinators (Fig. 3). We observed the prevalence of Asteraceae species ( 47 species) with this wide spectrum of flower visitors. Among them, $30.4 \%$ presented an extremely generalist pollination system with four or more flower visitor groups. Asteraceae, Verbenaceae, Apiaceae, and Myrtaceae can be cited as examples of this pollination system. However, there was a significant difference in this generalisation between plant families (Fig. 4). Asteraceae and Fabaceae presented a wide generalised pattern, while Orchidaceae and Solanaceae presented different pollinator groups at the family level but with a high degree of specialisation at the species level.

South Brazilian grasslands presented a great number of species visited by more than three groups of pollinators compared to other grassland plant-communities in South America. Most species from Venezuela and Mendoza (AR) grassland communities were pollinated by one or two groups, with few species visited by four groups. In contrast, South Brazilian grasslands and Bocaina grasslands (BR) had a similar frequency of extremally generalist species, pollinated by four distinct groups of pollinators (Fig. 5).

\section{Pollinator groups}

Bees were the most important and diversified pollinator group in the South Brazilian grasslands. Apis mellifera was recorded in 59 plant species from different plant families, while native bees were observed as potential pollinators of 130 species. Five subfamilies were found in our survey (Apinae - bumblebees: 4 spp.; carpenter bees: 11 spp.; oilcollecting bees: 11 spp.; stingless bees: 15 spp.; and others: 6 spp.; Andreninae: 13 spp.; Colletinae: 20 spp.; Megachiliinae: 15 spp.; and Halictinae: 50 spp.).

Few species were exclusively pollinated by groups other than bees. Plant species pollinated exclusively by beetles accounted for $10.7 \%$, while $7.1 \%$ were pollinated by wasps, $6 \%$ by flies, $6 \%$ by hawkmoths, $4.8 \%$ by butterflies, and $3.6 \%$ by hummingbirds (Fig. 3 ). The plant species pollinated exclusively by hawkmoths belonged to the families Solanaceae (two species), Orchidaceae (three species) and Cactaceae (one species). Plant species pollinated by wasps belonged to four different plant families, namely Apiaceae, Apocynaceae, Asteraceae, and Fabaceae. Four plant species were pollinated exclusively by butterflies, with one species belonging to Asteraceae, one to Fabaceae, and two species 

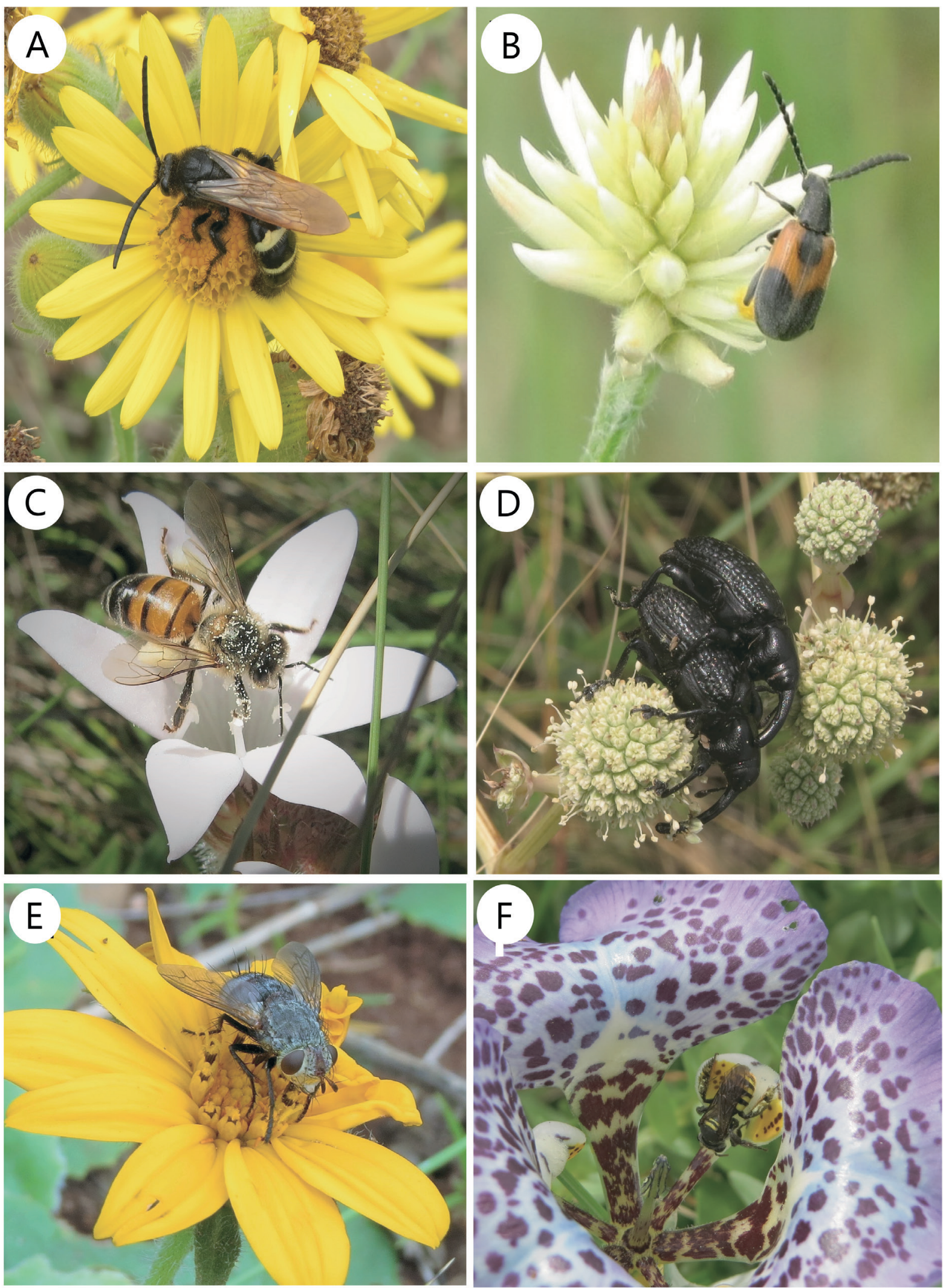

Figure 1. Some interactions registered in South Brazilian Grasslands: A, Campsomeris sp. (wasp) visiting Senecio sp.; B, a beetle visiting flowers of Pffafia tuberosa; C, Apis mellifera visiting flowers of Richardia grandiflora; $\mathbf{D}$, beetles (Curculionidae) visiting Eryingium horridum; E, a fly (Tachinidae) visiting Aspillia montevidensis and F, the oil-collecting bee Arhysoceble picta foraging oil in Kelissa brasiliensis. Interactions from A to E were recorded in Eldorado do Sul, Rio Grande do Sul, Brazil (2016) and F was from São Gabriel, Rio Grande do Sul, Brazil (2019). 
to Orchidaceae, and hummingbirds were the sole flower visitors of only three plant species.

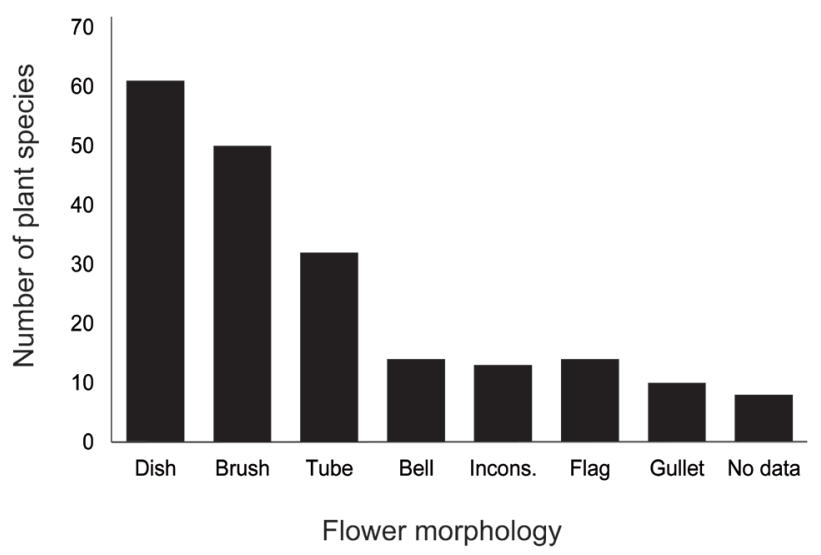

Figure 2. Number of plant species per flower type. Note that Asteraceae and Verbenaceae species are counted here as brush and dish, according to the morphology of the inflorescence.

\section{Discussion}

\section{Floral traits}

The prevalence of easily accessible flowers in this review had a similar pattern in many other plant communities from Brazilian high-altitude grasslands (Freitas \& Sazima 2006) to non-grassy ecosystems, such as the Caatinga in Brazil (Machado and Lopes 2004), and from herbaceous Mediterranean communities (Bosch et al. 1997) to Alpine communities (Makrodimos et al. 2008) in Europe. Both nectar and pollen were the most common floral rewards, observed in $53.6 \%$ of our sampled species, corroborating this common trait in most flowering plants (Galetto 2007). The same pattern was observed in Southeastern Brazil (Bocaina grasslands) (Freitas \& Sazima 2006). However, no reference, regardless of flower species, was made by Freitas \& Sazima (2006), while in the South Grasslands, we found five Orchidaceae species (3.3\% of the plant set) besides the female flowers of Begonia cucullata Willd., representing sexual mimicry (Avila Jr. et al. 2017). Regarding oil-flower species, we only found a similar frequency of oil-flowers in the Caatinga domain (Machado \& Lopes 2004).

\section{Pollination systems}

Some plant families were evaluated regarding the prevalence of pollination systems. Iridaceae and Cactaceae, except Cereus hildmannianus, were strongly associated with bee pollination. Iridaceae in South Brazilian grasslands comprehend several oil-producing flowers, such as the genera Herbertia Sweet, Cypella Herb, Sisyrinchium L. and Kelissa Ravenna, which are associated with oil and pollen collecting bees (Oleques et al. 2020). This prevalence of the bee-pollination system contrasted with Iridaceae assemblages in South Africa, for example, where besides bees there are plants pollinated by sunbirds, longue-tongued flies (Goldblatt \& Manning 2006). This plant group could, therefore, be an interesting model to verify the different efficiencies of both bee groups in the plant reproductive outputs.

Another predominantly bee-pollinated plant group was the family Cactaceae. The state of Rio Grande do Sul is one of the most important diversity centres of cacti in South America (Silva et al. 2011). The genus Parodia (besides Gymnocalycium, Frailea and Opuntia) presents strong convergence in floral traits, such as yellowish corolla and numerous stamens with high amounts of pollen as floral
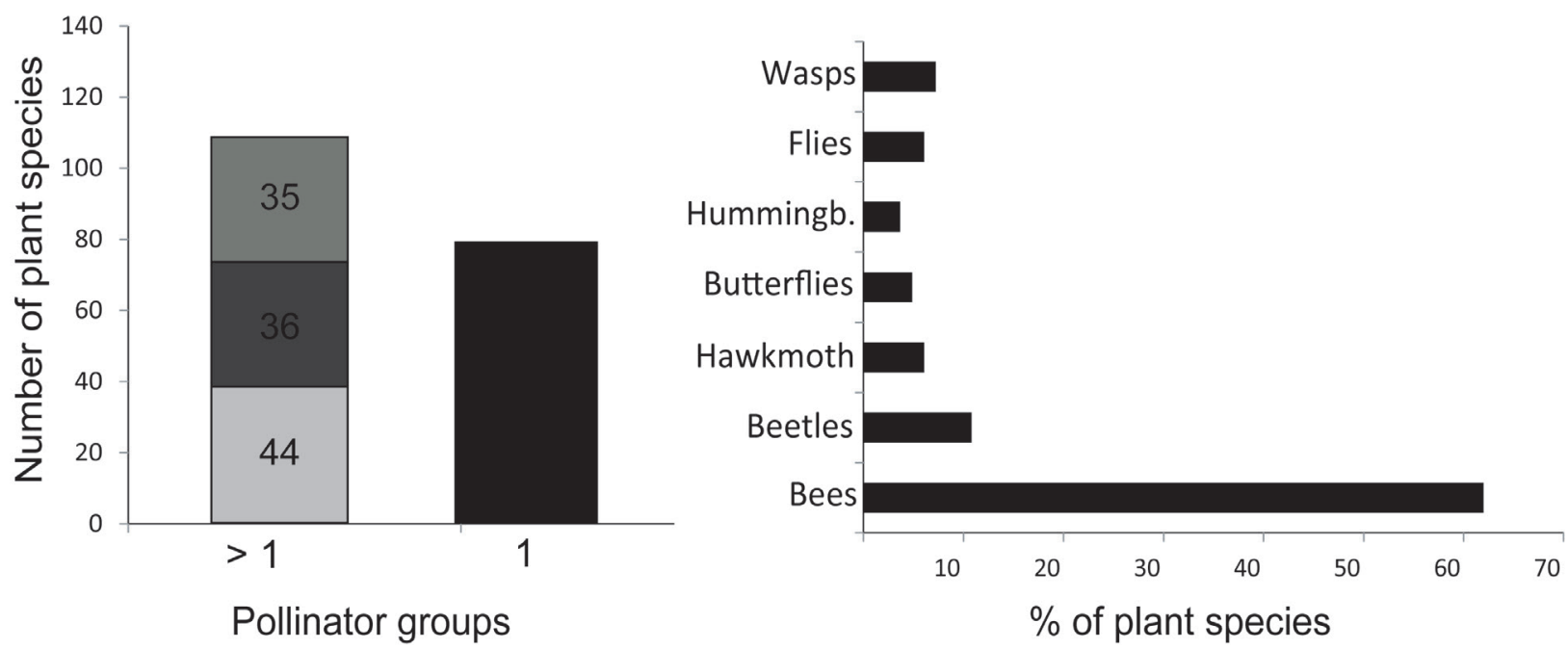

Figure 3. Number of species visited by more than one and by a single pollinator group (left). The numbers inside the bars (distinct shades of grey) are indicating the number of plant species pollinated by two, three and more than three groups (from bottom to up). On the right, the percentage of plant species visited only by bees, beetles, hawkmoths, butterflies, hummingbirds, flies and wasps. 

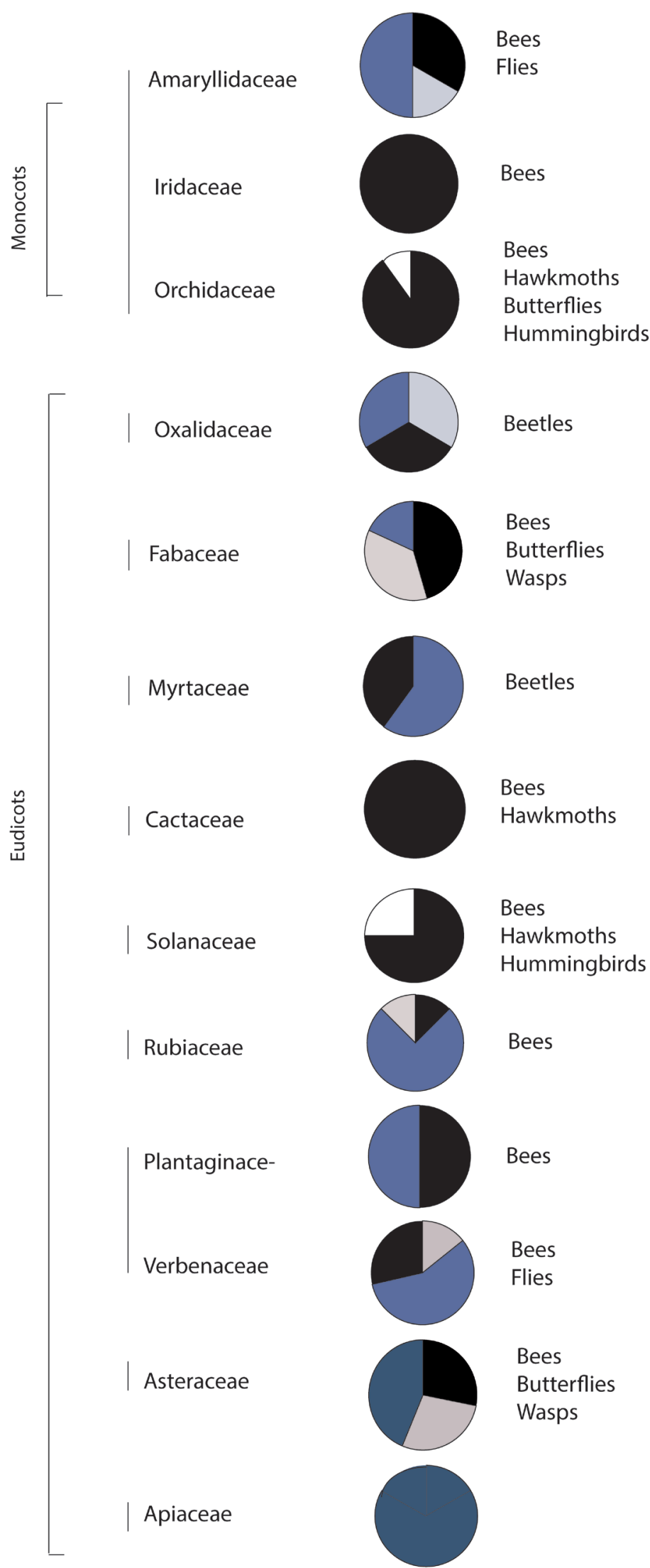

Insect specialist

Insect generalist (2 groups)

Insect generalist ( 3 or more)

Hawkmoths $\quad \square$ Vertebrate specialist

Figure 4. Frequency of different pollination systems within plant families in South Brazilian Grasslands (plant families with more than three species presented in this review). The pollinator groups described in the side of the graphs refers to specialized system cases observed in each plant family. 


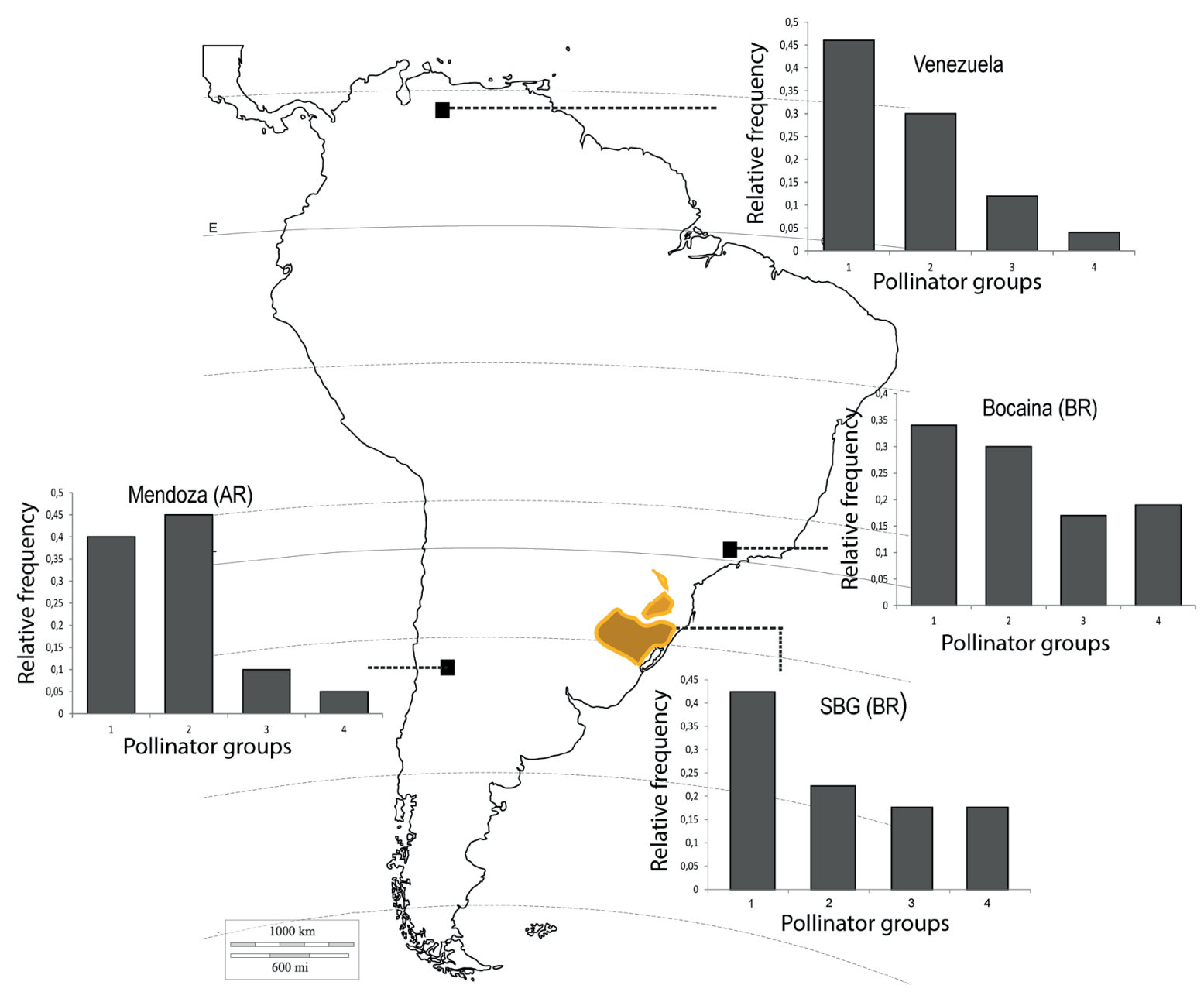

Figure 5. Relative frequency of plant species visited by one, two, three and four pollinator groups in distinct grassland communities of South America: Venezuela (Ramirez 2004), Mendoza (Vázquez 2007) and Southeastern Brazil (Freitas \& Sazima 2006). The yellow area in the map is indicating South Brazilian Grassland from Rio Grande do Sul, Santa Catarina and Paraná states in Brazil.

resources to several bee species. Its' wide corolla ensures that bees of different sizes collect pollen (from the small Dialictus to the large Cephalocolletes bipunctata, observed in Parodia crassigibba, for example). An interesting phenomenon in this cacti group is stamen movement (thigmonastic stamen), which ensures pollen deposition by these bees (Schlindwein \& Wittmann 1997). In this plant group, we may observe a wide spectrum of bee pollinators to extremely bee-specialised pollination systems (Cerceau et al. 2019). Furthermore, in this genus, there are some interesting cases of bee copulation behaviour during Opuntia flower visits, where male bees forage for females visiting flowers (Oliveira \& Schlindwein 2010). Plantaginaceae is another oil-producing flower group that, together with Iridaceae and Malpighiaceae, is exclusively pollinated by bees. Although some studies recorded other floral visitors (beetles and flies in Scoparia dulcis), the prevalence of bee species acting as potential pollinators was highlighted. This fact probably results from the kind of reward offered to pollinators.

Mixed pollination systems, with more than one single pollinator group, characterised most plant species in the
South grasslands. The prevalence of Asteraceae species could contribute to this pattern with this wide spectrum of flower visitors, which agrees with previous studies in South America that report the high importance of this plant group as a key resource for different guilds of pollinators (Torres \& Galetto 2008; Antonini \& Martins 2003; Freitas \& Sazima 2006, Pinheiro et al. 2008; Martins et al. 2013; Oleques et al. 2017). Those species generally have numerous flowers per inflorescence and present floral traits that make them accessible and attractive to a broad range of flower visitors, such as small floral tube size and secondary pollen presentation (Torres \& Galetto 2002; Antonini \& Martins 2003; Lunau et al. 2018). Moreover, Asteraceae is the most diverse plant group in the South Brazilian grasslands (excluding Poaceae) (Boldrini 1997; Overbeck 2007; Andrade et al. 2019) and could be considered crucial to the maintenance of many plant-pollinator interactions in grassland communities (Pinheiro et al. 2008; Oleques et al. 2019).

While most plant families presented wide generalisation spread in its species (i.e., the most of species presenting 
diverse pollinator groups), some other generalist plant groups presented a generalisation pattern but with some specialisation degree in pollination niches at the species level (Solanaceae and Orchidaceae, for example). This could be an interesting aspect of diversification associated with pollinator pressure along the evolutionary history of these plant groups. These species are good models to test the effective role of pollination strategies promoting diversification in grassland ecosystems (Gómez et al. 2015).

Freitas \& Sazima (2006), studying plant-pollinator interactions in Bocaina highland grasslands (Southeastern Brazil), found a similar pattern with a high prevalence of plants with two or more groups visiting flowers. However, these typical high-altitude grasslands presented higher equitability among pollination system frequencies (Freitas \& Sazima 2006) compared to our findings. Contrasting this pattern, in grasslands of extreme northern South America (Venezuela), Ramirez (2004) found a very high percentage of plants with only one group of floral visitors. Although with an overall lower number of specialised plant species than plants with generalist systems, the proportion between specialised-generalised pollination systems was quite similar in Venezuela, with a low number of extremely generalist plants (Ramirez 2004). The same proportion was observed in grasslands of Mendoza (Argentina), with an overall prevalence of pollination systems with more than two pollinator groups but with a low prevalence of extreme generalists (Vázquez 2007). Generalisation in pollination systems could be favoured by various ecological factors, such as unpredictability of the most important pollinator promoted by spatiotemporal variability in the pollinator assemblage, similarities among pollinators as selective agents, and geographical variations in the pollinator fauna along latitudinal gradients (Ollerton et al. 2006). Tropical areas generally present a larger number of potential pollinator groups that could promote higher specialisation, contrasting with generalisation systems in subtropical and temperate regions. However, this is not a pattern concerning plant-pollinator interactions (Ollerton \& Cranmer 2002), and plant-pollinator interactions in grasslands can reinforce this aspect. Grasslands from both extreme northern and Southern South America presented a similar frequency of plant species, with pollinators belonging to one pollinator group, however, with the prevalence of plants pollinated by two or more groups.

\section{Pollinator groups}

Our findings point out the importance of bees as pollinators of species in the South grasslands of Brazil. Bees are considered the most important and dominant pollinators in most plant communities (Proctor et. al. 1996). They are related to plant species with different floral traits, and their behaviour could vary according to their nutritional requirements, type of reward collected, and habitat (Stallman 2011; Giannini et al. 2012). Native stingless bees play an important role as pollinators of species occurring in the state of Rio Grande do Sul, and several species are considered oligolectic (Schlindwein 1998). Furthermore, stingless bees are known for their positive influence on the pollination of canola crop yields in Southern Brazil (Halinsk et al. 2018). The most diverse bee subfamily was Halictinae, being related to 66 plant species from 26 plant families. Although $A$. mellifera has been recorded as a visitor in several plant species, our finds elucidate the great diversity of native bees and their importance as pollinators of grassland plant species. All plants pollinated exclusively by hawkmoths present floral traits compatible with sphingophily, such as nocturnal anthesis, white/greenish colour, and nectar secreted in a tube or spur (Herrera 1996). The low prevalence of sphingophilous plant species in this review could reflects the rarity of studies on this pollination system in the South grasslands, mainly the absence of nocturnal observations during field work in community and species level.

The plant species pollinated by wasps presented morphologically generalised flowers; however, the unspecialised floral morphology could indicate the existence of other filters to exclude other pollinators, such as chemical characteristics of scent and nectar (Johnson and Steiner 2000). Specialised interactions between plants and wasps are typically associated with sexually deceptive or foodbased mimicry systems. Besides being uncommon, there are examples of rewarding plants, including pollination by vespids in Oxypetalum spp. and Blepharodon nitidus (Vell.) J.F.Macbr. (both milkweeds) in South America (Vieira and Shepherd 1999).

Few species were exclusively pollinated by butterflies because specialisation in pollination by butterflies is rarely found in plants (Johnson \& Bond 1994). Among all species visited exclusively by butterflies, only Epidendrum fulgens Brongn. presented floral traits considered adapted to butterfly pollination. In the last few years, there was an increase in studies of butterfly diversity in the South Brazilian grasslands. However, data about plant species pollinated by butterflies are still scarce and new studies on pollination ecology of species visited by this group are promising (Paz et al. 2013; Carvalho et al. 2015).

Species pollinated by hummingbirds share conspicuous reddish flowers, an important floral trait of bird pollination systems. Besides, colour, UV reflectance, nectar and scent were considered important traits to pollinators differentiation in Petunia spp (Rodrigues et al. 2018). Regarding to floral reward, except for Cattleya coccinea Lindl. (rewardless orchid), hummingbirds pollinated plants as Nicotiana forgetiana Hemsl., and Petunia exserta Stehmann, secret nectar is stored in a long corolla tube. Pollination by hummingbirds is a highly frequent pollination system in other plant communities studied in Brazil. However, most of the studied species are concentrated in forests of Southeastern Brazil (Buzatto et al. 2000; Canela \& Sazima 2005; Vizentin-Bugoni et al. 2014; Lunau et al. 2011). 


\section{Conclusion}

In conclusion, our review points out the lack of studies of plant-pollinator interactions in South Brazilian grasslands, mainly concerning some attributes of plants, such as nocturnal long-tube flowers pollinated by hawkmoths. In addition, there are no studies about the importance or efficiency of flies and beetles as pollinators of this particular vegetation. This is especially concerning in the Pampa domain because of the small extent of effectively protected areas and accelerated conversion of natural areas into extensive soybean monocultures in the last decade. Furthermore, an insufficient number of studies about mutualistic interactions and reproductive biology could compromise our understanding of plants and pollinators at risk. The prevalence of plants visited by more than three groups of pollinators highlight the generalist aspects of the interactions, which could be the result of the great diversity of plants with generalist flower traits, such as Asteraceae species. Based on our findings, bees are the most important group of pollinators related with both generalist and specialist plant groups, such as Asteraceae and Iridaceae. The nature of the information provided in this review is an important source of data that could be used to further pollination niche studies to understand the diversification and maintenance of South Brazilian grassland flora.

\section{Acknowledgements}

SSO is thankful to the Programa de Pós-Graduação em Botânica (UFRGS) and Coordenação de Aperfeiçoamento de Pessoal de Nivel Superior (CAPES) for the financial support and institutional scholarship - Finance Code 001. TTSC acknowledge CNPq by the fellowship received [grant number: 304506/2016-8].

\section{References}

Alves-dos-Santos I. 1997. Melittophilous plants, their pollen and flower visiting bees in Southern Brazil: 3. Pontederiaceae. Biociências, Porto Alegre 5: 3-18.

Armbruster WS. 2017. The specialization continuum in pollination systems: diversity of concepts and implications for ecology, evolution and conservation. Functional Ecology 31: 88-100.

Ando T, Nomura M, Tsukahara J, Watanabe H, et al. 2001. Reproductive Isolation in a Native Population of Petunia sensu Jussieu (Solanaceae). Annals of Botany 3: 403-413.

Andrade BO, Bonilha CL, Overbeck GE, et al. 2019. Classification of South Brazilian grasslands: Implications for conservation. Applied Vegetation Science 22: 168-184.

Antonini Y, Matins RP. 2003. The value of tree species (Caryocar brasiliense) for a stingless bee Melipona quadrifasciata quadrifasciata. Journal of Insect Conservation 3: 164-167.

APG IV - The Angiosperm Phylogeny Group. 2016. The linnean society of London. Botanical Journal of the Linnean Society 181: 1-20.

Avila Jr RS, Oleques SS, Marciniak B, Ribeiro JRI. 2017. Effects of modelmimic frequency on insect visitation and plant reproduction in a self-mimicry pollination system. AoB Plants 9: plx044. doi: 10.1093/ aobpla/plx044

Batalha MA. 2011. O cerrado não é um bioma. Biota Neotropica 11: 21-24. Beal-Neves M, Ely CV, Esteves MW, et al. 2020. The influence of urbanization and fire disturbance on plant-floral visitor mutualistic networks. Diversity 12: 141. doi:10.3390/d12040141.

Becker R. 2020. Polinização e biologia reprodutiva em Cereus hildmannianus K. Schum. e Pereskia aculeata Mill. (CACTACEAE). MSc Thesis, Universidade Federal do Rio Grande do Sul, Porto Alegre.

Bencke GA. 2009. Diversidade e conservação da fauna dos campos do sul do Brasil. In: Pillar VP. (ed.) Campos Sulinos - conservação e uso sustentável da biodiversidade. Brasília, Ministério do Meio Ambiente. p. 101-121.

Blochtein B, Nunes-Silva P, Halinski R, Lopes LA, Witter S. 2014. Comparative study of the floral biology and of the response of productivity to insect visitation in two rapeseed cultivars (Brassica napus L.) in Rio Grande do Sul. Brazilian Journal of Biology 74: 787794.

Boldrini II. 1997. Campos do Rio Grande do Sul: caracterização fisionômica e problemática ocupacional. Boletim do Instituto de BiociênciasUFRGS 56: 1-39.

Bosch J, Retana J, Cerdá X. 1997. Flowering phenology, floral traits and pollinator composition in a herbaceous Mediterranean plant Community. Oecologia 109: 583-591.

Buzatto S, Sazima M, Sazima I. 2000. Hummingbird-Pollinated Floras at Three Atlantic Forest Sites. Biotropica 32: 824- 841.

Caballero-Villalobos L, Silva-Arias GA, Buzatto C, Nervo MH, Singer RB. 2017. Generalized food-deceptive pollination in four Cattleya (Orchidaceae: Laeliinae) species from Southern Brazil. Flora 234: 195-206.

Canela MBF, Sazima M. 2005. The pollination of Bromelia antiacantha (Bromeliaceae) in Southeastern Brazil: Ornithophilous versus Melittophilous Features. Plant Biology 7: 411-416.

Carvalho APS, Piovesan G, Moraes ABB. 2015. Butterflies (Lepidoptera: Papilionoidea) of grassland areas in the Pampa biome, southern Brazil. Check List 11: 1772. doi: 10.15560/11.5.1772

Cerceau I, Siriani-Oliveira S, Dutra AL, Oliveira R, Schlindwein C. 2019. The cost of fidelity: foraging oligolectic bees gather huge amounts of pollen in a highly specialized cactus-pollinator association. Biological Journal of the Linnean Society 128: 30-43.

Corbet SA. 2006. A Typology of Pollination Systems: Implications for Crop Management and Conservation of Wild Plants. In: Waser NM, Ollerton J. (eds.) Plant-pollinator interactions: from specialization to generalization. Chicago, University of Chicago Press. p. 309-315. Coutinho LM. 2006. O conceito de bioma. Acta Botanica Brasilica 20: 1-11. Costa RACV, Morais ABB. 2008. Fenologia e visitantes florais de Erythrina crista-galli L. (Leguminosae: Faboideae) em Santa Maria, Rio Grande do Sul, Brasil. Biotemas 21: doi: 10.5007/2175-7925.2008v21n2p51

Faegri K, Van Der Pijl L. 1979. The principles of pollination ecology. New York, Pergamon Press.

Fenster CB, Armbruster WS, Wilson P, Dudash MR, Thomson JD. 2004. Pollination syndromes and floral specialization. Annual Review of Ecology, Evolution, and Systematics 35: 375-403.

Flora do Brasil 2020. 2020. Jardim Botânico do Rio de Janeiro. http:// floradobrasil.jbrj.gov.br/. 1 Aug. 2020.

Freitas L, Sazima M. 2006. Pollination biology in tropical high-altitude grassland in Brazil: Interactions at the community level. Annals of the Missouri Botanical Garden 93: 465-516.

French KE, Tkacz A, Lindsay AT. 2017. Conversion of grassland to arable decreases microbial diversity and alters community composition. Applied Soil Ecology 110: 43-52.

Galetto L. 2007. A systematic survey of floral nectar. In: Nicolson SW, Nepi M, Pacini E. (eds.) Nectaries and nectar. Dordrecht, Springer. p. 19-128.

García D, Hódar JA, Zamora R, Gómez JM.1996. Experimental study of pollination by ants in Mediterranean high mountain and arid habitats. Oecologia 105: 236-242.

Garibaldi LA, Cavalheiro LG, Vaissière BE, et al. 2016. Mutually beneficial pollinator diversity and crop yield outcomes in small and large farms. Science 351: 388-391. 
Giannini TC, Acosta AL, Garófalo CA, et al. 2012. Pollination services at risk: Bee habitats will decrease owing to climate change in Brazil. Ecological Modelling 244: 127-131.

Goldblatt P, Manning JC. 2006. Radiation of pollination systems in the Iridaceae of sub-Saharan Africa. Annals of Botany 97: 317-344.

Gómez JM, Perfectti F, Lorite J. 2015. The role of pollinators in floral diversification in a clade of generalist flowers. Evolution 69: 863-878.

Halinski R. dos Santos, CF, Kaehler TG, Blochtein B. 2018. Influence of Wild Bee Diversity on Canola Crop Yields. Sociobiology 65: 751-759.

Herrera CM. 1996. Floral traits and plant adaptation to insect pollinators: a devil's advocate approach. In: Lloyd DG, Barret SCH. (eds.) Floral biology: studies on floral evolution in animal pollinated plants. New York, Chapman \& Hall. p. 65-87.

Ippolito A, Fernandes GW, Holtsford TP. 2004. Pollinator preferences for Nicotiana alata, N. forgetiana, and their f1 hybrids. Evolution 58: 2634-2644.

Johnson SD, Bond WJ. 1994. Red flowers and butterfly pollination in the fynbos of South Africa. In: Arianoutsou M, Groves RH. (eds.) Plantanimal interactions in Mediterranean-type ecosystems. Dordrecht, Springer. p. 137-148.

Johnson SD, Steiner KE. 2000. Generalization versus specialization in plant pollination systems. Tree 15: 140-143.

Johnson SD. 2010. The pollination niche and its role in the diversification and maintenance of the southern African flora. Philosophical Transactions of the Royal Society B 365: 499-516.

Kearns CA, Inouye DW, Waser NM. 1998. Endangered Mutualisms: The Conservation of Plant-Pollinator Interactions. Annual Review of Ecology and Systematics 29: 83-112.

Lôbo C, Stefenon VM. 2018. Floral morphometric analysis of Prosopis affinis Spreng. (Fabaceae) suggests flexibility of the reproductive system in isolated populations within the Brazilian Pampa. Annals of Forest Research 61: 37-47.

Lopes BPTL. 2017. Aspectos morfológicos e ecológicos da ginodioicia em Dasyphyllum brasiliense (spreng.) Cabrera (Barnadesioideae, Asteraceae). MSc Thesis, Universidade Federal de Santa Maria, Santa Maria.

Lunau K, Papiorek S, Eltz T, Sazima M. 2011. Avoidance of achromatic colours by bees provides a private niche for hummingbirds. Journal of Experimental Biology 214: 1607-1612.

Lunau K, An L, Donda M, Hohmann M, Sermon L, Stegmanns V. 2018. Limitations of learning in the proboscis reflex of the flower visiting syrphid fly Eristalis tenax. PLOS ONE 13: e0194167. doi: 10.1371/ journal.pone. 0194167

Machado IC, Lopes AV. 2004. Floral traits and pollination systems in the Caatinga, a Brazilian Tropical Dry Forest. Annals of Botany 94: 365-376.

Makrodimos N, Blionis GJ, Krigas N, Vokou D. 2008. Flower morphology, phenology and visitor patterns in an alpine community on $\mathrm{Mt}$ Olympos, Greece. Flora 203: 449-468.

Maia FR, Telles FJ, Goldemberg R. 2018. Time and space affect reproductive biology and phenology in Tibouchina hatschbachii (Melastomataceae), and endemic shrub from subtropical grasslands of Southern Brazil. Botanical Journal of the Linnean Society 187: 689-703.

Martins AC, Aguiar AJC, Alves-dos-Santos I. 2013. Interaction between oil-collecting bees and seven species of Plantaginaceae. Flora 208: 401-411.

Memmott J, Craze PG, Waser MN, Price MV. 2007. Global warming and the disruption of plant-pollinator interactions. Ecology Letters 10: 710-717.

Mouga DMD, Noble CF, Bussmann DBG, Grug C. 2012. Bees and Plants in a transition area between Atlantic Rain Forest and Araucaria Forest in Southern Brazil. Revue d' Ecologie 67: 313-327.

Nunes-Silva P, Rosa DMJ, Witter S, et al. 2016. Visitantes Florais e Potenciais Polinizadores da Cultura da Macieira. Comunicado Técnico Embrapa 184. Available in: https://ainfo.cnptia.embrapa.br/digital/ bitstream/item/144651/1/Comunicado-Tecnico-184.pdf

Oleques SS, Overbeck GE, Avila Jr R. 2017. Flowering phenology and plant-pollinator interactions in a grassland community of Southern Brazil. Flora 229: 141-146.
Oleques SS, Radaeski JN, Bauerman S, Chauveau O, Souza-Chies TT. 2020. The specialization-generalization continuum in oil-bee pollination systems: a case study of six Brazilian species of Tigridieae (Iridaceae). Biological Journal of the Linnean Society 129: 701-716.

Oleques SS, Vizentin-Bugoni J, Overbeck GE. 2019. Influence of grazing intensity on patterns and structuring processes in plant-pollinator networks in a subtropical grassland. Arthropod-Plant Interactions 13: 757-770.

Oliveira R, Schlindwein C. 2010. Experimental demonstration of alternative mating tactics of male Ptilothrix fructifera (Hymenoptera Apidae). Animal Behaviour 80: 241-247.

Ollerton J, Cranmer L. 2002. Latitudinal trends in plant-pollinator interactions: are tropical plants more specialised? Oikos 98: 340350.

Ollerton J, Johnson SD, Hingston A, Waser N. 2006. Geographical variation in diversity and specificity of pollination systems. In: Waser $\mathrm{N}$, Ollerton J. (eds). Plant-pollinator interactions: from specialization to generalization. Chicago, Illinois, University of Chicago Press. p. 283-308.

Overbeck GE, Muller C, Fidelis A, et al. 2007. Brazil's neglected biome: The South Brazilian Campos. Perspectives in Plant Ecology, Evolution and Systematics 9: 101-116.

Overbeck GE, Müller S, Pillar VD, Pfadenhauer J. 2006. Floristic composition, environmental variation and species distribution patterns in burned grassland in southern Brazil. Brazilian Journal of Biology 66: 1073-1090.

Paz ALG, Ramanowisk HP, Morais ABB. 2013.Distribution of Satyrini (Lepidoptera, Nymphalidae) in Rio Grande do Sul State, southern Brazil. Ecological Research 28: 417-426.

Pedron M, Buzatto CR, Singer RB, et al. 2012. Pollination biology of four sympatric species of Habenaria (Orchidaceae: Orchidinae) from southern Brazil. Botanical Journal of the Linnean Society 170: 141156.

Pinheiro M, Abrão BE, Harter-Marques B, Miotto STS. 2008.Floral resources used by insects in a grassland community in Southern Brazil. Brazilian Journal of Botany 31: 469-489.

Pinto CE, Schlindwein C. 2014. Pollinator sharing and low pollen-ovule ratio diminish reproductive success in two sympatric species of Portulaca (Portulacaceae). Studies on Neotropical Fauna and Environment 50: 4-13.

Potts SG, Biesmeijer J, Kremen K, Neumann P, Scheweiger O, Kunin W. 2010. Global pollinator declines: Trends, impacts and drivers. Trends in Ecology \& Evolution 25: 345-53.

Proctor M, Yeo P, Lack A. 1996. The Natural History of Pollination. London, HarperCollins Publishers.

Ramírez N. 2004. Pollination specialization and time of pollination on a tropical Venezuelan plain: variations in time and space. Botanical Journal of the Linnean Society 145: 1-16.

Rodrigues DM, Caballero-Villalobos L, Turchetto C, Jacques RA, Kulehmeier C, Freitas LB. 2018. Do we truly understand pollination syndromes in Petunia as much as we suppose? AoB Plants 10: ply057. doi: 10.1093/ aobpla/ply057.

REBIPP - Rede Brasileira de Interações Planta- Polinizador. 2018. Relatório Temático sobre Polinização, Polinizadores e Produção de Alimentos no Brasil. Brasília, DF, Plataforma Brasileira de Biodiversidade e Serviços Ecossistêmicos. https://www.bpbes.net.br/wp-content/ uploads/2019/03/BPBES_CompletoPolinizacao-2.pdf.

Sanguinetti A, Buzatto CR, Pedron M, et al. 2012. Floral features, pollination biology and breeding system of Chloraea membranacea Lindl. (Orchidaceae: Chloraeinae). Annals of Botany 110: 1607-1621.

Sargent RD, Ackerly DD. 2008. Plant-pollinator interactions and the assembly of plant communities. Trends in Ecology \& Evolution 23: 123-30.

Schlindwein C, Wittmann D. 1995. Specialized solitary bees as effective pollinators of South Brazilian species of Notocactus and Gymnocalycium (Cactaceae). Bradleya 13: 25-34.

Schlindwein C, Wittmann D. 1997. Stamen movements in flowers of Opuntia (Cactaceae) favour oligolectic pollinators. Plant Systematics and Evolution 204: 179-193. 
Schlindwein C. 1998. Frequent oligolecty characterizing a diverse bee-plant community in a xerophytic bushland of Subtropical Brazil. Studies on Neotropical Fauna and Environment 33: 46-59.

Schlindwein C, Martins CF. 2000. Competition between the oligolectic bee Ptilothrix plumata (Anthophoridae) and the flower closing beetle Pristimerus calcaratus (Curculionidae) for floral resources of Pavonia cancellata (Malvaceae). Plant Systematics and Evolution 224: 183-194.

Silva SR. 2011. Plano de Ação Nacional Para a Conservação das Cactáceas. Brasília, ICMBio. https://www.icmbio.gov.br/portal/faunabrasileira/ plano-de-acao-nacional-lista/148-pan-cactaceas. 1 Aug. 2020.

Simmons BI, Wauchope HS, Amano T, Dicks LV, Sutherland WJ, Dakos V. 2020. Estimating the risk of species interaction loss in mutualistic communities. PLOS Biology 18: e3000843. doi: 10.1371/journal. pbio. 3000843

Siriani-Oliveira S, Cerceau I, Schlindwein C. 2019. Specialized protagonists in a plant-pollinator interaction: the pollination of Blumenbachia insignis (Loasaceae). Plant Biology 22: 167-176.

Siriani-Oliveira S, Oliveira R, Schlindwein C. 2018. Pollination of Blumenbachia amana (Loasaceae): flower morphology and partitioned pollen presentation guarantee a private reward to a specialist pollinator. Biological Journal of the Linnean Society 124: 479-491.

Stallman HR. 2011. Ecosystem services in agriculture: Determining suitability for provision by collective management. Ecological Economics 71: 131-139.

Streher N, Guerra E, Lüdtke R, Semir J, Dutlh JHA. 2018. Selfincompatibility in Habranthus gracilifolius (Amaryllidaceae): pre- and post-pollination barriers. Brazilian Journal of Botany 41: 375-384.

Torres C, Galetto L. 2008. Importancia de los polinizadores en la reproducción de Asteraceae de Argentina Central. Acta Botánica Venezuelica 31: 473-494.
Torres C, Galetto L. 2011. Flowering phenology of co-occurring Asteraceae: a matter of climate, ecological interactions, plant attributes or of evolutionary relationships among species? Organisms Diversity \& Evolution 11: 9-19.

Torres C, Galetto L. 2002. Are Nectar Sugar Composition and Corolla Tube Length Related to the Diversity of Insects that Visit Asteraceae Flowers? Plant Biology 4: 360-366.

Valiente-Banuet A, Aizen M, Alcántara JM, et al. 2015. Beyond species loss: extinction of interactions in a changing world. Functional Ecology 29: 299-307.

Vázquez D, Milián CJ, Williams NM, Blüthgen N, et al. 2007. Species abundance and asymmetric interaction strength in ecological networks. Oikos 116: 1120-1127.

Vieira MF, Shepherd GJ. 1999. Pollinators of Oxypetalum (Asclepiadaceae) IN southeastern Brazil. Revista Brasileira de Biologia 59: 693-704.

Vizentin-Bugoni J, Maruyama PK, Sazima M. 2014. Processes entangling interactions in communities: forbidden links are more important than abundance in a hummingbird-plant network. Proceedings of the Royal Society B: Biological Sciences 281: 20132397. doi: 10.1098/ rspb.2013.2397

Weiner CN, Werner M, Linsenmair KE, Blüthgen N. 2014. Land-use impacts on plant-pollinator networks: interaction strength and specialization predict pollinator declines. Ecology 95: 466-474.

Witter S, Blochtein B, Nunes-Silva P, et al. 2014. The bee community and its relationship to canola seed production in homogenous agricultural áreas. Journal of Pollination Ecology 12: 15-21.

Wolowski M, Carvalheiro LG, Freitas L. 2017. Influence of plant-pollinator interactions on the assembly of plant and hummingbird communities. Journal of Ecology 105: 332-344. 